\title{
A note on the Fröhlich dynamics in the strong coupling limit
}

\author{
David Mitrouskas ${ }^{1,2}$
}

Received: 26 March 2020 / Revised: 25 February 2021 / Accepted: 3 March 2021 /

Published online: 5 April 2021

(C) The Author(s) 2021

\begin{abstract}
We revise a previous result about the Fröhlich dynamics in the strong coupling limit obtained in Griesemer (Rev Math Phys 29(10):1750030, 2017). In the latter it was shown that the Fröhlich time evolution applied to the initial state $\varphi_{0} \otimes \xi_{\alpha}$, where $\varphi_{0}$ is the electron ground state of the Pekar energy functional and $\xi_{\alpha}$ the associated coherent state of the phonons, can be approximated by a global phase for times small compared to $\alpha^{2}$. In the present note we prove that a similar approximation holds for $t=O\left(\alpha^{2}\right)$ if one includes a nontrivial effective dynamics for the phonons that is generated by an operator proportional to $\alpha^{-2}$ and quadratic in creation and annihilation operators. Our result implies that the electron ground state remains close to its initial state for times of order $\alpha^{2}$, while the phonon fluctuations around the coherent state $\xi_{\alpha}$ can be described by a time-dependent Bogoliubov transformation.
\end{abstract}

Keywords Fröhlich polaron · Strong coupling limit · Effective dynamics · Quantum corrections

Mathematics Subject Classification 81Q05 $\cdot 81 \mathrm{Q} 15 \cdot 82 \mathrm{C} 10$

\section{Introduction and main result}

\subsection{The model}

The Fröhlich polaron is a quantum model for a large polaron which describes an electron in an ionic lattice interacting with the excitations (phonons) of this lattice $[1,12]$. Large refers to the assumption that the extension of the electron is much larger compared to the lattice spacing which can thus be approximated by a continuum. In

$\bowtie$ David Mitrouskas

mitrouskas@mathematik.uni-stuttgart.de ; david.mitrouskas@ist.ac.at

1 Fachbereich Mathematik, Universität Stuttgart, Stuttgart, Germany

2 Present Address: Institute of Science and Technology Austria (IST Austria), Am Campus 1, 3400 Klosterneuburg, Austria 
this model, the energy and the dynamics of the electron and the phonons are described by the Fröhlich Hamiltonian

$$
H_{\mathrm{phys}, \alpha}^{\mathrm{F}}=p^{2} \otimes 1+1 \otimes N+\sqrt{\alpha} \phi\left(G_{x}\right)
$$

that acts on the Hilbert space $\mathscr{H}=L^{2}\left(\mathbb{R}^{3}, \mathrm{~d} x\right) \otimes \mathcal{F}$. Here $\mathcal{F}=\bigoplus_{n=0}^{\infty} L^{2}\left(\mathbb{R}^{3}, \mathrm{~d} k\right)^{\otimes_{\text {sym }}^{n}}$ is the bosonic Fock space, $x$ and $p=-i \nabla_{x}$ denote the position and momentum operator of the electron, respectively, and $N$ is the number operator on $\mathcal{F}$. The interaction between the electron and the phonons is described by $\phi\left(G_{x}\right)=a\left(G_{x}\right)+a^{*}\left(G_{x}\right)$ with $a(f)$ and $a^{*}(f)$ the usual annihilation and creation operators on $\mathcal{F}$ and $G_{x}$ the bounded multiplication operator defined for any $x \in \mathbb{R}^{3}$ by the function

$$
G_{x}(k)=\frac{e^{-i k x}}{2 \pi|k|}
$$

The creation and annihilation operators satisfy the canonical commutation relations

$$
\begin{aligned}
{\left[a(f), a^{*}(g)\right] } & =\langle f, g\rangle_{L^{2}}, \\
{[a(f), a(g)] } & =\left[a^{*}(f), a^{*}(g)\right]=0 \quad \forall f, g \in L^{2}\left(\mathbb{R}^{3}, \mathrm{~d} k\right) .
\end{aligned}
$$

Finally the number $\alpha>0$ is a dimensionless coupling parameter that models the strength of the interaction. The regime $\alpha \rightarrow \infty$ is called the strong coupling limit.

By a change of units which corresponds to rescaling all lengths by a factor $\alpha^{-1}$, the Fröhlich Hamiltonian $H_{\text {phys, }}^{\mathrm{F}}$ is unitarily equivalent to the operator $\alpha^{2} H_{\alpha}^{\mathrm{F}}$ with ${ }^{1}$

$$
H_{\alpha}^{\mathrm{F}}=p^{2} \otimes 1+1 \otimes \alpha^{-2} N+\alpha^{-1} \phi\left(G_{x}\right) .
$$

In the analysis of the strong coupling limit it is more convenient to work in strong coupling units, i.e., to use $H_{\alpha}^{\mathrm{F}}$ instead of the original Fröhlich Hamiltonian $H_{\mathrm{phys}, \alpha}^{\mathrm{F}}$ and then consider rescaled values of energy $E=\alpha^{-2} E_{\text {phys }}$ and time $t=\alpha^{2} t_{\text {phys }}$. This explains why $t=O\left(\alpha^{2}\right)$ is the time scale we are interested in for the dynamics generated by $H_{\alpha}^{\mathrm{F}}$.

In this work we study the large $\alpha$ limit of the time-evolved state $\Psi_{\alpha}(t)=e^{-i H_{\alpha}^{\mathrm{F}} t} \Psi_{\alpha}$ for a special initial state, namely the Pekar product state $\Psi_{\alpha}=\varphi_{0} \otimes \xi_{\alpha}$ where $\varphi_{0} \in$ $H^{1}\left(\mathbb{R}^{3}, \mathrm{~d} x\right)$ is the self-trapped electron ground state of the Pekar energy functional (to be defined below) and $\xi_{\alpha}=W\left(\alpha f_{0}\right)^{*} \Omega_{0}$ is the corresponding coherent phonon state. That is to say, $\Omega_{0}=(1,0,0, \ldots)$ is the normalized vacuum state in $\mathcal{F}$ and

$$
W\left(\alpha f_{0}\right)=\exp \left(a^{*}\left(\alpha f_{0}\right)-a\left(\alpha f_{0}\right)\right)
$$

denotes the Weyl operator w.r.t. the function

$$
\alpha f_{0}(k)=\alpha\left\langle\varphi_{0}, G .(k) \varphi_{0}\right\rangle_{L^{2}}=\frac{\alpha}{2 \pi|k|} \int_{\mathbb{R}^{3}} e^{-i k x}\left|\varphi_{0}(x)\right|^{2} \mathrm{~d} x .
$$

\footnotetext{
${ }^{1}$ See [13, Appendix B].
} 
We recall that the Weyl operator is unitary and satisfies the shift relation

$$
W\left(\alpha f_{0}\right)^{*} a(g) W\left(\alpha f_{0}\right)=a(g)+\alpha\left\langle g, f_{0}\right\rangle_{L^{2}}
$$

for any $g \in L^{2}\left(\mathbb{R}^{3}, \mathrm{~d} k\right)$.

The Pekar energy functional is defined by

$$
\mathcal{E}^{\mathrm{P}}(\varphi)=\int_{\mathbb{R}^{3}}|\nabla \varphi(x)|^{2} \mathrm{~d} x-\frac{1}{2} \int_{\mathbb{R}^{3}} \int_{\mathbb{R}^{3}} \frac{|\varphi(x)|^{2}|\varphi(y)|^{2}}{|x-y|} \mathrm{d} x \mathrm{~d} y
$$

with constraint $\|\varphi\|_{L^{2}}=1$. It was shown in [20] that $\mathcal{E}^{\mathrm{P}}(\varphi)$ admits a unique minimizer (unique up to spatial translations)

$$
\varphi_{0} \in H^{1}\left(\mathbb{R}^{3}, \mathrm{~d} x\right) \cap\left\{\varphi \in L^{2}\left(\mathbb{R}^{3}, \mathrm{~d} x\right):\|\varphi\|_{L^{2}}=1\right\}
$$

that can be chosen positively. The minimizer further solves the Euler-Lagrange equation $\left(h^{\varphi_{0}}-\lambda\right) \varphi_{0}=0$ where

$$
h^{\varphi_{0}}=p^{2}+V^{\varphi_{0}}, \quad V^{\varphi_{0}}(x)=-2 \operatorname{Re}\left\langle G_{x}, f_{0}\right\rangle_{L^{2}},
$$

and $\lambda=\mathcal{E}^{\mathrm{P}}\left(\varphi_{0}\right)-\left\|f_{0}\right\|_{L^{2}}^{2}$. By its positivity, it follows that $\varphi_{0}$ is the unique ground state of the Schrödinger operator $h^{\varphi_{0}}$ and that $\lambda=\inf \sigma\left(h^{\varphi_{0}}\right)$ belongs to the discrete spectrum of $h^{\varphi_{0}}$, see [25, Sec. 12]. Introducing the orthogonal projector $Q=1-P$ with $P=\left|\varphi_{0}\right\rangle\left\langle\varphi_{0}\right|$, we hence know that $h^{\varphi_{0}}-\lambda$ is a positive operator when restricted to the closed subspace $\operatorname{ran} Q \subseteq L^{2}\left(\mathbb{R}^{3}, \mathrm{~d} x\right)$. This allows the definition of the restricted resolvent

$$
R=Q\left(h^{\varphi_{0}}-\lambda\right)^{-1} Q
$$

as a bounded operator in $L^{2}\left(\mathbb{R}^{3}, \mathrm{~d} x\right)$. The fact that $R$ is independent of $\alpha$ and thus bounded uniformly as $\alpha \rightarrow \infty$ is a crucial ingredient in the analysis of the strong coupling limit of $\Psi_{\alpha}(t)$. In a nutshell, it ensures a separation of scales as $\alpha \rightarrow \infty$ of the different parts of the Fröhlich Hamiltonian $H_{\alpha}^{\mathrm{F}}$ when the latter is applied to states of the form $\varphi \otimes W\left(\alpha f_{0}\right)^{*} \eta$ for suitable $\varphi \in \operatorname{ran} Q$ and $\eta \in \mathcal{F}$.

That the scale separation of the different parts in $H_{\alpha}^{\mathrm{F}}$ allows an effective description of the Fröhlich dynamics for times $t=o\left(\alpha^{2}\right)$ was first observed in [13]. There, it was shown that the wave function $\Psi_{\alpha}(t)=e^{-i H_{\alpha}^{\mathrm{F}} t} \varphi_{0} \otimes W\left(\alpha f_{0}\right)^{*} \Omega_{0}$ remains close to its initial state up to a global phase factor, i.e.,

$$
\left\|\Psi_{\alpha}(t)-e^{-i \mathcal{E}^{\mathrm{P}}\left(\varphi_{0}\right) t} \varphi_{0} \otimes W\left(\alpha f_{0}\right)^{*} \Omega_{0}\right\|_{\mathscr{H}} \leq C|t|^{1 / 2} \alpha^{-1}
$$

for some $C>0$. Since the initial state is normalized to one, the upper bound is meaningful for $t \ll \alpha^{2}$. A similar approximation was obtained in [19] for more general initial states, namely Pekar product states in which the electron is initially trapped in the classical field produced by a given coherent state of the phonons. Modulo 
a global phase factor, the effective dynamics is then described by the Pekar product state $\varphi_{\mathrm{LP}}(t) \otimes W\left(\alpha f_{\mathrm{LP}}(t)\right)^{*} \Omega_{0}$ with $\left(\varphi_{\mathrm{LP}}(t), f_{\mathrm{LP}}(t)\right)$ solving the time-dependent Landau-Pekar equations, cf. [19, Eqn. (8)]. In fact, the effective dynamics in (12) can be understood as the special case in which $\left(\varphi_{0}, f_{0}\right)$ are the stationary ground state solutions of the Landau-Pekar equations. The proof of the nonstationary problem, however, is technically more demanding as it is based on a nonlinear adiabatic theorem for the solution of the Landau-Pekar equations, see [19, Theorem II.1]. Loosely speaking, the latter shows that the scale separation of the different parts in the Fröhlich Hamiltonian remains valid on some suitable time scale also in the nonstationary case. An adiabatic theorem for the Landau-Pekar equations in one spatial dimension has been derived in $[9,10]$. Earlier results about the Fröhlich dynamics in the strong coupling limit provide approximations for $t=o(\alpha)$ but for much more general initial Pekar product states $\varphi \otimes W(\alpha f)^{*} \Omega_{0}$ with no particular assumption about the relation between $\varphi$ and $f$, see $[7,8]$. To our knowledge, there are no results available to date that provide an approximation for the Fröhlich dynamics for $t=O\left(\alpha^{2}\right) .^{2}$

Remark 1 The particular choice of our initial state $\Psi_{\alpha}=\varphi_{0} \otimes \xi_{\alpha}$ is motivated by Pekar's approximation of the ground state energy of the Fröhlich Hamiltonian [24]. Taking the expectation value of $H_{\alpha}^{\mathrm{F}}$ for general Pekar states $\varphi \otimes W(\alpha f)^{*} \Omega_{0}$ and minimizing over the phonon mode $f \in L^{2}(\mathbb{R}, \mathrm{d} k)$ leads to the Pekar functional $\mathcal{E}^{\mathrm{P}}(\varphi)$. That Pekar's approximations is accurate in the strong coupling limit was rigorously proved in [3] and later, using a different approach which provided in addition a quantitative error estimate, in [21]. They showed

$$
\inf \sigma\left(H_{\alpha}^{\mathrm{F}}\right)=\mathcal{E}^{\mathrm{P}}\left(\varphi_{0}\right)+o(1)
$$

as $\alpha \rightarrow \infty$. The physical picture behind this result is that the electron creates a classical phonon field which in turn leads to an effective trapping of the electron. This self-trapping mechanism is described by the ground state of (8). Let us also mention that the rigorous derivation of the next order contribution in (13) is still an open problem that was recently solved in [11] for a model in which the Fröhlich polaron is assumed to be confined to a suitably bounded region $\Lambda \subset \mathbb{R}^{3}$.

Remark 2 We note that (4), and equally (1), is somewhat formal since $G_{x} \notin$ $L^{2}\left(\mathbb{R}^{3}, \mathrm{~d} k\right)$ and hence $\phi\left(G_{x}\right)$ is not a densely defined operator. However, by a wellknown argument that goes back to Lieb and Yamazaki [22], the right side of (4) defines a closed semi-bounded quadratic form with domain given by the form domain of $p^{2} \otimes 1+1 \otimes N$. The Hamiltonian $H_{\alpha}^{\mathrm{F}}$ is then defined as the unique self-adjoint operator associated with this quadratic form, cf. [26, Thm. VIII.15]. For the purpose of this work, it is sufficient to use the form representation given in (4). Alternative approaches to define the Fröhlich Hamiltonian with an explicit characterization of its domain have been discussed more recently in [14,17].

\footnotetext{
2 After the submission of this article new results have been reported about the derivation of the LandauPekar equations (and the corresponding quantum fluctuations) for times of order $\alpha^{2}[6,18]$.
} 


\subsection{Effective dynamics}

Our goal is to derive an approximation similar to (12) for times $t=O\left(\alpha^{2}\right)$. To achieve this, we compare $\Psi_{\alpha}(t)$ with an effective time evolution that is generated by the Hamiltonian

$$
H_{\alpha}^{\varphi_{0}}=1 \otimes\left\langle\varphi_{0},\left(H_{\alpha}^{\mathrm{F}}-\left(\alpha^{-1} \phi(G .)-V^{\varphi_{0}}\right)(R \otimes 1)\left(\alpha^{-1} \phi(G .)-V^{\varphi_{0}}\right)\right) \varphi_{0}\right\rangle_{L^{2}}
$$

acting on $\mathscr{H}=L^{2}\left(\mathbb{R}^{3}, \mathrm{~d} x\right) \otimes \mathcal{F}$. This operator acts nontrivially only on the Fock space. The expectation value in the second factor of the tensor product is taken with respect to the inner product in $L^{2}\left(\mathbb{R}^{3}, \mathrm{~d} x\right)$. Equivalently we could write the second factor as a partial trace,

$$
\operatorname{Tr}_{L^{2}\left(\mathbb{R}^{3}\right)}\left(\left|\varphi_{0}\right\rangle\left\langle\varphi_{0}\left|\left(H_{\alpha}^{\mathrm{F}}-\left(\alpha^{-1} \phi(G .)-V^{\varphi_{0}}\right)(R \otimes 1)\left(\alpha^{-1} \phi(G .)-V^{\varphi_{0}}\right)\right)\right| \varphi_{0}\right\rangle\left\langle\varphi_{0}\right|\right)
$$

In the following proposition we clarify the difference compared to the ansatz in (12) and, more importantly, we obtain the existence of a unitary time evolution generated by $H_{\alpha}^{\varphi_{0}}$.

Proposition 1.1 For any $\alpha>0$ we have

$$
W\left(\alpha f_{0}\right) H_{\alpha}^{\varphi_{0}} W\left(\alpha f_{0}\right)^{*}-\mathcal{E}^{\mathrm{P}}\left(\varphi_{0}\right)=1 \otimes \alpha^{-2}\left(N-A^{\varphi_{0}}\right)
$$

with the operator $A^{\varphi_{0}}: \mathcal{F} \rightarrow \mathcal{F}$ defined by

$$
\begin{aligned}
A^{\varphi_{0}} & =\left\langle\varphi_{0}, \phi(G .)(R \otimes 1) \phi(G .) \varphi_{0}\right\rangle_{L^{2}} \\
& =\int_{\mathbb{R}^{3}} \int_{\mathbb{R}^{3}}\left\langle\varphi_{0}, G .(k) R G .(l) \varphi_{0}\right\rangle_{L^{2}}\left(a_{k}^{*}+a_{-k}\right)\left(a_{l}^{*}+a_{-l}\right) d k d l
\end{aligned}
$$

Moreover, $\mathscr{D}(N) \subseteq \mathscr{D}\left(N-A^{\varphi_{0}}\right)$ and $N-A^{\varphi_{0}}$ is essentially self-adjoint on $\mathcal{F}$. (We denote its closure again by $N-A^{\varphi_{0}}$.)

We prove this proposition in Sect. 2.4. By unitarity of the Weyl operator, it follows that $H_{\alpha}^{\varphi_{0}}$ is self-adjoint on $\mathscr{H}$ and thus $\exp \left(-i H_{\alpha}^{\varphi_{0}} t\right)$ defines a unitary time evolution.

Let us emphasize that the effective Hamiltonian acts nontrivially only on the phonons. This implies in particular that the time evolved state $\exp \left(-i H_{\alpha}^{\varphi_{0}} t\right) \varphi_{0} \otimes \xi_{\alpha}$ is still an exact product. Because of the operator $A^{\varphi_{0}}$ in (16), however, the coherent state structure of the initial state $\xi_{\alpha}$ is not conserved. In this regard, our effective dynamics is different compared to the known results discussed in the previous section.

Remark 3 As a motivation of our ansatz in (14) let us mention its analogy to the well-known second-order perturbation formula

$$
E_{\varepsilon}=\left\langle u_{0},\left(H_{\varepsilon}-\varepsilon V R_{0} \varepsilon V\right) u_{0}\right\rangle+O\left(\varepsilon^{3}\right) \quad(\varepsilon \ll 1)
$$


for the nondegenerate ground state energy $E_{\varepsilon}$ of a suitable Hamiltonian $H_{\varepsilon}=$ $H_{0}+\varepsilon V$ by means of the ground state vector $u_{0}$ of $H_{0}$ and the reduced resolvent $R_{0}=\left(1-\left|u_{0}\right\rangle\left\langle u_{0}\right|\right)\left(H_{0}-\left\langle u_{0}, H_{0} u_{0}\right\rangle\right)^{-1}\left(1-\left|u_{0}\right\rangle\left\langle u_{0}\right|\right)$. Despite this analogy, we emphasize that the expectation value in (14) is taken only w.r.t. to the electron wave function $\varphi_{0} \in L^{2}\left(\mathbb{R}^{3}, \mathrm{~d} x\right)$ and not w.r.t. to the full Pekar product $\varphi_{0} \otimes \xi_{\alpha}$. The reason why the expectation value w.r.t. $\varphi_{0} \otimes \xi_{\alpha}$ would not lead to a good ansatz for the effective dynamics is the appearance of the factor $\alpha^{-2}$ in front of the number operator $N$.

\subsection{Main results}

We are now ready to state our main results.

Theorem 1.2 Let $\varphi_{0} \in H^{1}\left(\mathbb{R}^{3}, d x\right)$ be the unique minimizer of the Pekar functional (8) with $\left\|\varphi_{0}\right\|_{L^{2}}=1$ and let $f_{0} \in L^{2}\left(\mathbb{R}^{3}, d k\right)$ be defined as in (6). Let further $\eta_{0} \in \mathcal{F}$ satisfy $\left\|\eta_{0}\right\|_{\mathcal{F}}=1$ and $\sup _{\alpha>0}\left\|(N+1)^{5 / 2} \eta_{0}\right\|_{\mathcal{F}}<\infty$. Then, there are constants $c, C>0$ such that

$$
\left\|\left(e^{-i H_{\alpha}^{\mathrm{F}} t}-e^{-i H_{\alpha}^{\varphi_{0}} t}\right) \varphi_{0} \otimes W\left(\alpha f_{0}\right)^{*} \eta_{0}\right\|_{\mathscr{H}} \leq C \alpha^{-1} \exp \left(c|t| \alpha^{-2}\right)
$$

for all $t \in \mathbb{R}$ and $\alpha>0$.

Since the initial state is normalized to one, the approximation is accurate for $t=O\left(\alpha^{2}\right)$ (indeed, it is accurate for $t \ll \alpha^{2} \ln \alpha$ ). As a direct consequence of (19) together with $\left[e^{-i H_{\alpha}^{\varphi_{0}} t}, P \otimes 1\right]=0$, we obtain the following statement that shows that the reduced density of the electron remains approximately constant.

Corollary 1.3 Under the same assumptions as in Theorem 1.2 there exist constants $c, C>0$ such that

$$
\left.\operatorname{Tr}_{L^{2}}\left|\operatorname{Tr}_{\mathcal{F}}\right| \Psi_{\alpha}(t)\right\rangle\left\langle\Psi_{\alpha}(t)|-| \varphi_{0}\right\rangle\left\langle\varphi_{0}|| \leq C \alpha^{-1} \exp \left(c|t| \alpha^{-2}\right)\right.
$$

with $\Psi_{\alpha}(t)=e^{-i H_{\alpha}^{\mathrm{F}} t} \varphi_{0} \otimes W\left(\alpha f_{0}\right)^{*} \eta_{0}$.

Theorem 1.2 shows that on the time scale $t=O\left(\alpha^{2}\right)$ it is important to include the creation and annihilation of noncoherent phonons in the effective time evolution. In earlier findings which provided approximations for $t=o(\alpha)[7,8]$ and $t=o\left(\alpha^{2}\right)$ $[13,19]$, respectively, it was not necessary to take such noncoherent phonons into account as the effective dynamics was still described by exact Pekar product states. In our next corollary, we use the fact that the operator $N-A^{\varphi_{0}}$ is quadratic in creation and annihilation operators in order to describe the fluctuations around the coherent phonons by means of a time-dependent Bogoliubov transformation.

To make the last statement precise we need to introduce some well-known notions related to the Bogoliubov transformation. The generalized annihilation and creation operators are defined by $A(F)=a(f)+a^{*}(g)$ and $A^{*}(F)=a^{*}(f)+a(g)$, respectively, for any $F=f \oplus J g \in L^{2}\left(\mathbb{R}^{3}, \mathrm{~d} k\right) \oplus L^{2}\left(\mathbb{R}^{3}, \mathrm{~d} k\right)$ where $J$ denotes 
the complex conjugation map $(J g)(x)=\overline{g(x)}$. A bounded invertible map $\mathcal{V}$ on $L^{2}\left(\mathbb{R}^{3}, \mathrm{~d} k\right) \oplus L^{2}\left(\mathbb{R}^{3}, \mathrm{~d} k\right)$ is called a Bogoliubov map if it satisfies

$$
A^{*}(\mathcal{V} F)=A(\mathcal{V} \mathcal{J} F), \quad\left[A(\mathcal{V} F), A^{*}(\mathcal{V} G)\right]=\langle F, \mathcal{S} G\rangle_{L^{2} \oplus L^{2}}
$$

for all $F, G \in L^{2}\left(\mathbb{R}^{3}, \mathrm{~d} k\right) \oplus L^{2}\left(\mathbb{R}^{3}, \mathrm{~d} k\right)$ where

$$
\mathcal{J}=\left(\begin{array}{cc}
0 & J \\
J & 0
\end{array}\right), \quad \mathcal{S}=\left(\begin{array}{cc}
1 & 0 \\
0 & -1
\end{array}\right)
$$

In case that the Bogoliubov map $\mathcal{V}$ is a Hilbert-Schmidt operator, i.e., if $\mathcal{V}^{*} \mathcal{V}$ is trace class, it can be implemented as a unitary operator on $\mathcal{F}$. This is the content of the ShaleStinespring condition which states that there exists a unitary operator $U_{\mathcal{V}}: \mathcal{F} \rightarrow \mathcal{F}$ such that

$$
U_{\mathcal{V}} A(F) U_{\mathcal{V}}^{*}=A(\mathcal{V} F)
$$

for any $F \in L^{2}\left(\mathbb{R}^{3}, \mathrm{~d} k\right) \oplus L^{2}\left(\mathbb{R}^{3}, \mathrm{~d} k\right)$ if and only if $\operatorname{Tr} \mathcal{V}^{*} \mathcal{V}<\infty$, see, e.g., [27, Thm. 9.5]. We call the operator $U_{\mathcal{V}}$ the Bogoliubov transformation associated with the Bogoliubov map $\mathcal{V}$. Finally we need the concept of (pure bosonic) quasi-free states in $\mathcal{F}$. A quasi-free state $\eta \in \mathcal{F}$ is defined by the property that there is a Bogoliubov map $\mathcal{V}_{\eta}$ such that $\eta$ can be written as the transformed vacuum $\eta=U_{\mathcal{V}_{\eta}} \Omega_{0}$ (in particular, $\Omega_{0}$ is quasi-free). For a detailed introduction to Bogoliubov transformations and quasi-free states, we refer to [27, Sec. 9 and 10].

Our next goal is to show that the dynamics of the noncoherent phonons in $\Psi_{\alpha}(t)=e^{-i H_{\alpha}^{\mathrm{F}} t} \varphi_{0} \otimes W\left(\alpha f_{0}\right)^{*} \eta_{0}$ can be described by a time-dependent Bogoliubov transformation $U_{\mathcal{V}_{\alpha}(t)}$ associated with the Bogoliubov map

$$
\mathcal{V}_{\alpha}(t)=\exp \left[-\frac{i t}{\alpha^{2}}\left(\begin{array}{cc}
1-\mathcal{G} & \mathcal{K} \\
-\overline{\mathcal{K}} & -1+\overline{\mathcal{G}}
\end{array}\right)\right] \mathcal{V}_{\alpha}(0), \quad \mathcal{V}_{\alpha}(0)=\left(\begin{array}{ll}
1 & 0 \\
0 & 1
\end{array}\right)
$$

where $\mathcal{K}, \mathcal{G}$ denote integral operators in $L^{2}\left(\mathbb{R}^{3}, \mathrm{~d} k\right)$ defined by the kernels

$$
\begin{aligned}
& \mathcal{K}(k, l)=(2 \pi|k|)^{-1}(2 \pi|l|)^{-1}\left\{\left\langle\varphi_{0}, e^{-i k x} R^{-i l x} \varphi_{0}\right\rangle_{L^{2}}+\left\langle\varphi_{0}, e^{-i l x} R^{-i k x} \varphi_{0}\right\rangle_{L^{2}}\right\}, \\
& \mathcal{G}(k, l)=(2 \pi|k|)^{-1}(2 \pi|l|)^{-1}\left\{\left\langle\varphi_{0}, e^{+i k x} \operatorname{Re}^{-i l x} \varphi_{0}\right\rangle_{L^{2}}+\left\langle\varphi_{0}, e^{-i k x} \operatorname{Re}^{+i l x} \varphi_{0}\right\rangle_{L^{2}}\right\},
\end{aligned}
$$

and where $\overline{\mathcal{K}}, \overline{\mathcal{G}}$ are to be understood as the integral operators with kernels $\overline{\mathcal{K}}(k, l)=$ $\overline{\mathcal{K}(k, l)}=\mathcal{K}(-k,-l)$ and $\overline{\mathcal{G}}(k, l)=\overline{\mathcal{G}(k, l)}=\mathcal{G}(l, k)$, respectively.

Corollary 1.4 Under the same assumptions as in Theorem 1.2 with the additional requirement that $\eta_{0} \in \mathcal{F}$ is quasi-free, there exist constants $c, C>0$ such that

$$
\left.\operatorname{Tr}_{\mathcal{F}}\left|\operatorname{Tr}_{L^{2}}\right| W\left(\alpha f_{0}\right) \Psi_{\alpha}(t)\right\rangle\left\langle W\left(\alpha f_{0}\right) \Psi_{\alpha}(t)|-| U_{\mathcal{V}_{\alpha}(t)} \eta_{0}\right\rangle\left\langle U_{\mathcal{V}_{\alpha}(t)} \eta_{0}||\right.
$$




$$
\leq C \alpha^{-1} \exp \left(c|t| \alpha^{-2}\right)
$$

with $\Psi_{\alpha}(t)=e^{-i H_{\alpha}^{\mathrm{F}} t} \varphi_{0} \otimes W\left(\alpha f_{0}\right)^{*} \eta_{0}$ and $U_{\mathcal{V}_{\alpha}(t)}$ the Bogoliubov transformation associated with the time-dependent Bogoliubov map $\mathcal{V}_{\alpha}(t)$ defined in (24).

The remainder of this note is organized as follows. We conclude section one with a short remark about the notation and a sketch of the proof of Theorem 1.2. In the second section we begin by stating two preliminary lemmas which are useful for the proof of Theorem 1.2. The latter is given in Sect. 2.2, whereas the preliminary lemmas are proved in Sect. 2.3. Finally we prove Proposition 1.1 together with Corollaries 1.3 and 1.4 in Sect. 2.4 .

\subsection{Notation}

From now on, we omit the tensor product with the identity in operators of the form $h^{\varphi_{0}}=h^{\varphi_{0}} \otimes 1$ and $N=1 \otimes N$. Moreover we make use of the abbreviation

$$
\delta G_{x}=G_{x}-f_{0},
$$

with $f_{0}$ defined as in (6) and by $\varphi_{0} \in H^{1}\left(\mathbb{R}^{3}, \mathrm{~d} x\right)$ we always denote the ground state of the Pekar functional (8) satisfying $\left\|\varphi_{0}\right\|_{L^{2}}=1$. The letter $C$ is used for positive constants that are independent of $t$ and $\alpha$. The exact value of $C$ may vary from line to line.

\subsection{Sketch of the proof}

The proof of Theorem 1.2 is motivated mainly by the proof of inequality (12) given in [13]. To demonstrate our main idea it is instructive to start with a sketch of the derivation of (12) (in slightly different way compared to [13]). To this end, we use the shift relation (7) to verify

$$
W\left(\alpha f_{0}\right) H_{\alpha}^{\mathrm{F}} W\left(\alpha f_{0}\right)^{*}-\mathcal{E}^{\mathrm{P}}\left(\varphi_{0}\right)=h^{\varphi_{0}}-\lambda+\alpha^{-2} N+\alpha^{-1} \phi(\delta G .) .
$$

With $W\left(\alpha f_{0}\right) e^{-i H_{\alpha}^{\mathrm{F}} t} W\left(\alpha f_{0}\right)^{*}=\exp \left(-i W\left(\alpha f_{0}\right) H_{\alpha}^{\mathrm{F}} W\left(\alpha f_{0}\right)^{*} t\right)$ and by Duhamel's principle, one then obtains

$$
\begin{aligned}
& \left\|\left(e^{-i H_{\alpha}^{\mathrm{F}} t}-e^{-i \mathcal{E}^{\mathrm{P}}\left(\varphi_{0}\right) t}\right) \varphi_{0} \otimes W\left(\alpha f_{0}\right)^{*} \Omega_{0}\right\|_{\mathscr{H}}^{2} \\
& \quad=-2 \alpha^{-1} \operatorname{Re} \int_{0}^{t} i\left\langle e^{-i\left(h^{\varphi}-\lambda+\alpha^{-2} N+\alpha^{-1} \phi(\delta G .)\right) s} \varphi_{0} \otimes \Omega_{0}, Q \phi(\delta G .) \varphi_{0} \otimes \Omega_{0}\right\rangle_{\mathscr{H}} \mathrm{d} s .
\end{aligned}
$$

Note that we further used $\left(h^{\varphi_{0}}-\lambda\right) \varphi_{0}=0$ and $\phi\left(\delta G_{x}\right) \varphi_{0} \otimes \Omega_{0}=Q \phi\left(\delta G_{x}\right) \varphi_{0} \otimes \Omega_{0}$ which holds because of $\left\langle\varphi_{0}, \delta G . \varphi_{0}\right\rangle_{L^{2}}=0$ (recall $P=\left|\varphi_{0}\right\rangle\left\langle\varphi_{0}\right|$ and $Q=1-P$ ). A rough estimate of the right side would now lead to an upper bound proportional 
to $|t| \alpha^{-1}$. The reason why the right side behaves actually better than this is a phase inside the integral which oscillates with nonzero ( $\alpha$-independent) frequency. ${ }^{3}$ To take advantage of this phase we rewrite the integrand as

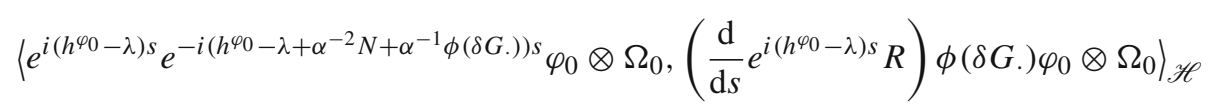

and then integrate by parts. This leads to a perturbation like expansion of (30) which among other contributions (e.g., the boundary terms which are of order $\alpha^{-1}$ ) includes the term

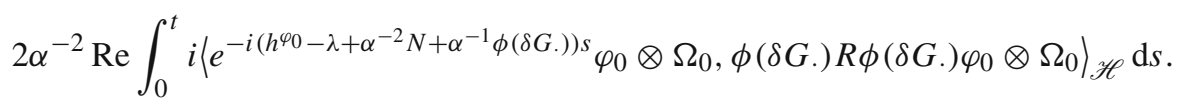

Apart from some technical difficulties being related to $G_{x} \notin L^{2}\left(\mathbb{R}^{3}, \mathrm{~d} k\right)$, one then applies the estimate (here we use that $R$ is uniformly bounded)

$$
\begin{aligned}
& \left|\left\langle e^{-i\left(h^{\varphi}-\lambda+\alpha^{-2} N+\alpha^{-1} \phi(\delta G .)\right) s} \varphi_{0} \otimes \Omega_{0}, \phi(\delta G .) R \phi(\delta G .) \varphi_{0} \otimes \Omega_{0}\right\rangle_{\mathscr{H}}\right| \\
& \quad \leq C\left\|(N+1) \Omega_{0}\right\|_{\mathcal{F}}
\end{aligned}
$$

in order to arrive at $|(32)| \leq C \alpha^{-2}|t|$. This bound is indeed the reason why (12) is limited to $t=o\left(\alpha^{2}\right)$. Our idea to improve upon this is to use the oscillating phase in (32) a second time. Inserting the identity $1=P+Q$ on the left of $\phi\left(\delta G_{x}\right)$ we obtain two contributions,

$$
=2 \alpha^{-2} \int_{0}^{t} \operatorname{Re} i\left\langle e^{\left.-i\left(h^{\varphi} 0-\lambda\right)+\alpha^{-2} N+\alpha^{-1} \phi(\delta G .)\right) s} \varphi_{0} \otimes \Omega_{0}, Q \phi(\delta G .) R \phi(\delta G .) \varphi_{0} \otimes \Omega_{0}\right\rangle_{\mathscr{C}} \mathrm{d} s,
$$

$$
=2 \alpha^{-2} \int_{0}^{t} \operatorname{Re} i\left(e^{\left.-i\left(h^{\varphi} 0-\lambda\right)+\alpha^{-2} N+\alpha^{-1} \phi(\delta G .)\right) s} \varphi_{0} \otimes \Omega_{0}, P \phi(\delta G .) R \phi(\delta G .) \varphi_{0} \otimes \Omega_{0}\right\rangle_{\mathscr{H}} \mathrm{d} s .
$$

In the first one we can proceed similarly as before and improve the bound through integration by parts to $|(32 . \mathrm{a})| \leq C\left(\alpha^{-2}+|t| \alpha^{-3}\right)$. In the second line, however, integration by parts is not applicable since $\left(h^{\varphi_{0}}-\lambda\right) P=0$. In other words, there is no fast oscillating phase in this term and thus (32.b) seems to be really of order $|t| \alpha^{-2}$. To avoid this term in the first place we include the operator $W\left(\alpha f_{0}\right)^{*} \alpha^{-2}\left(N-A^{\varphi_{0}}\right) W\left(\alpha f_{0}\right)$ into the effective dynamics, see (16). Starting over again with the new effective dynamics we now obtain an additional term in the first-order Duhamel expansion which cancels exactly the contribution from (32.b), cf. (51) and (57). Because of the nontrivial

$\overline{3}$ One should think of the improved $t$-dependence in $\int_{0}^{t} i e^{i b s} \mathrm{~d} s=b^{-1}\left(e^{i b t}-1\right)$ compared to $\int_{0}^{t} 1 \mathrm{~d} s=t$. 
dynamics of the phonons we now have to take into account the number of excitations in the effective time evolution. Using a Gronwall argument, this is shown to be bounded by a constant times $\exp \left(c|t| \alpha^{-2}\right)$ which leads to the exponential factor in (19). This already explains much of our proof and aside from the technical details, it would lead to an upper bound in (19) that is proportional to $\alpha^{-1 / 2} \exp \left(|t| \alpha^{-2}\right)$. By a third integration by parts we can improve the accuracy of this upper bound further and finally arrive at the stated bound in (19).

In principle there is no obstacle for continuing the explained strategy by additional integration by parts. In the present work, however, we do not pursue this idea further for the following two reasons. First, the effective Hamiltonian would become more complicated and involve terms with more than two creation and annihilation operators (hence the advantage of having a quadratic Hamiltonian would be lost). Second, an extension of our result to even longer times (e.g., $t=O\left(\alpha^{3}\right)$ ) would not follow as an immediate consequence. This is due to the difficulty of controlling the number of phonon excitations produced in the effective time evolution. In Lemma 2.2 we shall see that our bound would be insufficient for times $t \gg \alpha^{2}$.

Remark 4 The described idea of improving the approximation to longer times by changing the effective Hamiltonian as in (16) was similarly used also in $[15,16]$. These works treat very different models, namely the dynamics of a single tracer particle resp. two tracer particles interacting with an ideal Fermi gas in the high density limit. The used approximations and the proofs of their accuracy, however, are analogous to the one we apply to the Fröhlich Polaron. The scale separation in these models comes from the large momenta of the gas modes that are close to the Fermi surface. (For the ideal Fermi gas, high density is equivalent to a large Fermi momentum.)

\section{Proofs}

\subsection{Preliminary lemmas}

Before we start with the proof of Theorem 1.2, let us state two lemmas with several helpful estimates. Their proofs are postponed to Sect. 2.3.

Lemma 2.1 Let $P=\left|\varphi_{0}\right\rangle\left\langle\varphi_{0}\right|$ and $R$ as defined in (11). There is a constant $C>0$ such that for any $\Psi=\varphi_{0} \otimes \eta \in \mathscr{H}$ with $\eta \in \mathscr{D}\left(N^{5 / 2}\right)$, the following bounds hold.

$$
\begin{aligned}
& \|R \phi(\delta G .) \Psi\|_{\mathscr{H}}+\|[N, R \phi(\delta G .)] \Psi\|_{\mathscr{H}} \leq C\left\|(N+1)^{1 / 2} \eta\right\|_{\mathcal{F}} \\
& \left\|(R \phi(\delta G .))^{2} \Psi\right\|_{\mathscr{H}}+\left\|\left[N,(R \phi(\delta G .))^{2}\right] \Psi\right\|_{\mathscr{H}} \leq C\|(N+1) \eta\|_{\mathcal{F}}, \\
& \left\|(R \phi(\delta G .))^{3} \Psi\right\|_{\mathscr{H}}+\left\|P \phi(\delta G .)(R \phi(\delta G .))^{2} \Psi\right\|_{\mathscr{H}} \\
& +\|R \phi(\delta G .) P \phi(\delta G .) R \phi(\delta G .) \Psi\|_{\mathscr{H}}+\left\|\left[N,(R \phi(\delta G .))^{3}\right] \Psi\right\|_{\mathscr{H}} \leq C\left\|(N+1)^{3 / 2} \eta\right\|_{\mathcal{F}},
\end{aligned}
$$

$\left\|(R \phi(\delta G .))^{2} P \phi(\delta G .) R \phi(\delta G .) \Psi\right\|_{\mathscr{H}} \leq C\left\|(N+1)^{2} \eta\right\|_{\mathcal{F}}$,

$\left\|(R \phi(\delta G .))^{3} P \phi(\delta G .) R \phi(\delta G .) \Psi\right\|_{\mathscr{H}} \leq C\left\|(N+1)^{5 / 2} \eta\right\|_{\mathcal{F}}$. 
Moreover for $\Phi \in H^{1}\left(\mathbb{R}^{3}, d x\right) \otimes \mathcal{F}$ we have

$$
\left|\left\langle\Phi, \phi(\delta G .)(R \phi(\delta G .))^{3} \Psi\right\rangle_{\mathscr{H}}\right| \leq C\left(1+\|p \Phi\|_{\mathscr{H}}\right)\left\|(N+1)^{2} \eta\right\|_{\mathcal{F}} .
$$

Lemma 2.2 Let $\eta \in \mathscr{D}\left(N^{5 / 2}\right)$ with $\|\eta\|_{\mathcal{F}}=1$ and $\sup _{\alpha>0}\left\|(N+1)^{5 / 2} \eta_{0}\right\|_{\mathcal{F}}<\infty$. Then there are constants $c, C>0$ such that

$$
\begin{gathered}
\sum_{j=0}^{5}\left\|(N+1)^{j / 2} \exp \left(-i \alpha^{-2}\left(N-A^{\varphi_{0}}\right) t\right) \eta\right\|_{\mathcal{F}}^{2} \leq C \exp \left(c|t| \alpha^{-2}\right) \\
\left\|p e^{-i H_{\alpha}^{\mathrm{F}} t} \varphi_{0} \otimes W\left(\alpha f_{0}\right)^{*} \eta\right\|_{\mathscr{H}} \leq C
\end{gathered}
$$

for all $t \in \mathbb{R}$ and $\alpha>0$.

\subsection{Proof of Theorem 1.2}

We recall the relations

$$
\begin{aligned}
W\left(\alpha f_{0}\right) H_{\alpha}^{\mathrm{F}} W\left(\alpha f_{0}\right)^{*}-\mathcal{E}^{\mathrm{P}}\left(\varphi_{0}\right) & =h^{\varphi_{0}}-\lambda+\alpha^{-2} N+\alpha^{-1} \phi\left(\delta G_{x}\right), \\
W\left(\alpha f_{0}\right) H_{\alpha}^{\varphi_{0}} W\left(\alpha f_{0}\right)^{*}-\mathcal{E}^{\mathrm{P}}\left(\varphi_{0}\right) & =\alpha^{-2}\left(N-A^{\varphi_{0}}\right),
\end{aligned}
$$

which are verified by the commutation relations

$$
\begin{aligned}
W\left(\alpha f_{0}\right) \alpha^{-2} N W\left(\alpha f_{0}\right)^{*} & =\alpha^{-2} N-\alpha^{-1} \phi\left(f_{0}\right)+\left\|f_{0}\right\|_{L^{2}}^{2}, \\
W\left(\alpha f_{0}\right) \alpha^{-1} \phi\left(G_{x}\right) W\left(\alpha f_{0}\right)^{*} & =\alpha^{-1} \phi\left(G_{x}\right)+V^{\varphi_{0}},
\end{aligned}
$$

which in turn are easily obtained via (7). Using the unitarity of the Weyl operator we thus shall estimate

$$
\begin{aligned}
& \left\|\left(e^{-i H_{\alpha}^{\mathrm{F}} t}-e^{-i H_{\alpha}^{\varphi_{0}} t}\right) \varphi_{0} \otimes W\left(\alpha f_{0}\right)^{*} \eta_{0}\right\|_{\mathscr{H}} \\
& \quad=\left\|\left(e^{-i\left(h^{\varphi_{0}}-\lambda+\alpha^{-2} N+\alpha^{-1} \phi(\delta G .)\right) t}-e^{-i \alpha^{-2}\left(N-A^{\varphi_{0}}\right) t}\right) \varphi_{0} \otimes \eta_{0}\right\|_{\mathscr{H}} .
\end{aligned}
$$

For notational convenience let us abbreviate

$$
\psi_{\alpha}(t)=e^{-i\left(h^{\varphi_{0}}-\lambda+\alpha^{-2} N+\alpha^{-1} \phi\left(\delta G_{x}\right)\right) t} \varphi_{0} \otimes \eta_{0}, \quad \xi_{\alpha}(t)=\varphi_{0} \otimes e^{-i \alpha^{-2}\left(N-A^{\varphi_{0}}\right) t} \eta_{0} .
$$

By the fundamental theorem of calculus we have

$$
\begin{aligned}
\left\|\psi_{\alpha}(t)-\xi_{\alpha}(t)\right\|_{\mathscr{H}}^{2} & =2 \operatorname{Re}\left\langle\psi_{\alpha}(0), \psi_{\alpha}(0)-e^{i\left(h^{\varphi_{0}}-\lambda+\alpha^{-2} N+\alpha^{-1} \phi(\delta G .)\right) t} \xi_{\alpha}(t)\right\rangle_{\mathscr{H}} \\
& =-2 \operatorname{Re}\left\langle\psi_{\alpha}(0), \int_{0}^{t} \frac{\mathrm{d}}{\mathrm{d} s}\left(e^{i\left(h^{\varphi}-\lambda+\alpha^{-2} N+\alpha^{-1} \phi(\delta G .)\right) s} \xi_{\alpha}(s)\right) \mathrm{d} s\right\rangle_{\mathscr{H}} .
\end{aligned}
$$


Hence we get

$$
\left\|\psi_{\alpha}(t)-\xi_{\alpha}(t)\right\|_{\mathscr{H}}^{2}=2 \operatorname{Re} f_{\alpha}(t)+2 \operatorname{Re} g_{\alpha}(t)
$$

with

$$
\begin{aligned}
& f_{\alpha}(t)=-i \alpha^{-1} \int_{0}^{t}\left\langle\psi_{\alpha}(s),\left.\phi(\delta G .) \xi_{\alpha}(s)\right|_{\mathscr{H}} \mathrm{d} s,\right. \\
& g_{\alpha}(t)=-i \alpha^{-2} \int_{0}^{t}\left\langle\psi_{\alpha}(s), P \phi(\delta G .) R \phi(\delta G .) \xi_{\alpha}(s)\right\rangle_{\mathscr{H}} \mathrm{d} s .
\end{aligned}
$$

Note that here we have used $\left[N-A^{\varphi_{0}}, P\right]=0, P \xi_{\alpha}(s)=\xi_{\alpha}(s)$ and $\left(h^{\varphi_{0}}-\lambda\right) P=0$. With $1=P+Q$ and $\left\langle\varphi_{0}, \delta G \cdot \varphi_{0}\right\rangle_{L^{2}}=0$ one further obtains

$$
f_{\alpha}(t)=-i \alpha^{-1} \int_{0}^{t}\left\langle\psi_{\alpha}(s),\left.Q \phi(\delta G .) \xi_{\alpha}(s)\right|_{\mathscr{H}} \mathrm{d} s .\right.
$$

In the first part of the proof we do three integrations by part w.r.t. the time variable $s$. This leads to a perturbation like expansion of (52) into different contributions. In particular, after the first partial integration, we obtain one term that equals $-g_{\alpha}(t)$. Since this term would contribute an error of order $|t| \alpha^{-2}$, it is crucial that we included the second-order correction in the effective dynamics. All remaining contributions will be estimated separately in the second part of the proof and finally lead to the error in (19).

To prepare the first integration by parts we use the restricted resolvent $R=Q\left(h^{\varphi_{0}}-\right.$ $\lambda)^{-1} Q$ in order to write

$$
f_{\alpha}(t)=-\alpha^{-1} \int_{0}^{t}\left\langle e^{i\left(h^{\varphi} 0-\lambda\right) s} \psi_{\alpha}(s),\left(\frac{\mathrm{d}}{\mathrm{d} s} e^{i\left(h^{\varphi} 0-\lambda\right) s} R\right) \phi(\delta G .) \xi_{\alpha}(s)\right\rangle_{\mathscr{H}} \mathrm{d} s .
$$

Using

$$
\begin{aligned}
\frac{\mathrm{d}}{\mathrm{d} s} e^{i\left(h^{\varphi_{0}}-\lambda\right) s} \psi_{\alpha}(s) & =-i e^{i\left(h^{\varphi_{0}}-\lambda\right) s}\left(\alpha^{-2} N+\alpha^{-1} \phi\left(\delta G_{x}\right)\right) \psi_{\alpha}(s), \\
\frac{\mathrm{d}}{\mathrm{d} s} \xi_{\alpha}(s) & =-i \alpha^{-2}\left(N-A^{\varphi_{0}}\right) \xi_{\alpha}(s),
\end{aligned}
$$

together with $R \psi_{\alpha}(0)=R \varphi_{0} \otimes \eta_{0}=0$, one finds by integration by parts

$$
\begin{aligned}
f_{\alpha}(t)= & -\alpha^{-1}\left\langle\psi_{\alpha}(t), R \phi(\delta G .) \xi_{\alpha}(t)\right\rangle_{\mathscr{H}} \\
& +i \alpha^{-3} \int_{0}^{t}\left\langle\psi_{\alpha}(s), R\left([N, \phi(\delta G .)]+\phi(\delta G .) A^{\varphi_{0}}\right) \xi_{\alpha}(s)\right\rangle_{\mathscr{H}} \mathrm{d} s \\
& +i \alpha^{-2} \int_{0}^{t}\left\langle\psi_{\alpha}(s), \phi(\delta G .) R \phi(\delta G .) \xi_{\alpha}(s)\right\rangle_{\mathscr{H}} \mathrm{d} s .
\end{aligned}
$$


In the last line the prefactor $\alpha^{-2}$ is not sufficient and we need to do a second integration by parts. For that, we insert again the identity $1=P+Q$ on the left of $\phi\left(\delta G_{x}\right)$. The term containing $P$ equals

$$
i \alpha^{-2} \int_{0}^{t}\left\langle\psi_{\alpha}(s), P \phi(\delta G .) R \phi(\delta G .) \xi_{\alpha}(s)\right\rangle_{\mathscr{H}} \mathrm{d} s=-g_{\alpha}(t)
$$

and thus

$$
(56 \mathrm{c})+g_{\alpha}(t)=i \alpha^{-2} \int_{0}^{t}\left\langle\psi_{\alpha}(s), Q \phi(\delta G .) R \phi(\delta G .) \xi_{\alpha}(s)\right\rangle_{\mathscr{H}} \mathrm{d} s .
$$

In this term we can integrate by parts similarly as in (53) which leads to

$$
\begin{aligned}
(56 \mathrm{c})+g_{\alpha}(t)= & \alpha^{-2}\left\langle\psi_{\alpha}(t), R \phi(\delta G .) R \phi(\delta G .) \xi_{\alpha}(t)\right\rangle_{\mathscr{H}} \\
& -i \alpha^{-4} \int_{0}^{t}\left\langle\psi_{\alpha}(s),[N, R \phi(\delta G .) R \phi(\delta G .)] \xi_{\alpha}(s)\right\rangle_{\mathscr{H}} \mathrm{d} s \\
& -i \alpha^{-4} \int_{0}^{t}\left\langle\psi_{\alpha}(s), R \phi(\delta G .) R \phi(\delta G .) A^{\varphi_{0}} \xi_{\alpha}(s)\right\rangle_{\mathscr{H}} \mathrm{d} s \\
& -i \alpha^{-3} \int_{0}^{t}\left\langle\psi_{\alpha}(s), P \phi(\delta G .) R \phi(\delta G .) R \phi(\delta G .) \xi_{\alpha}(s)\right\rangle_{\mathscr{H}} \mathrm{d} s \\
& -i \alpha^{-3} \int_{0}^{t}\left\langle\psi_{\alpha}(s), Q \phi(\delta G .) R \phi(\delta G .) R \phi(\delta G .) \xi_{\alpha}(s)\right\rangle_{\mathscr{H}} \mathrm{d} s .
\end{aligned}
$$

In the last line we do a third integration by parts, i.e.,

$$
\begin{aligned}
(59 \mathrm{e})= & -\alpha^{-3}\left\langle\psi_{\alpha}(t), R \phi(\delta G .) R \phi(\delta G .) R \phi(\delta G .) \xi_{\alpha}(t)\right\rangle_{\mathscr{H}} \\
& +i \alpha^{-5} \int_{0}^{t}\left\langle\psi_{\alpha}(s),[N, R \phi(\delta G .) R \phi(\delta G .) R \phi(\delta G .)] \xi_{\alpha}(s)\right\rangle_{\mathscr{H}} \mathrm{d} s \\
& +i \alpha^{-5} \int_{0}^{t}\left\langle\psi_{\alpha}(s), R \phi(\delta G .) R \phi(\delta G .) R \phi(\delta G .) A^{\varphi_{0}} \xi_{\alpha}(s)\right\rangle_{\mathscr{H}} \mathrm{d} s \\
& +i \alpha^{-4} \int_{0}^{t}\left\langle\psi_{\alpha}(s), \phi(\delta G .) R \phi(\delta G .) R \phi(\delta G .) R \phi(\delta G .) \xi_{\alpha}(s)\right\rangle_{\mathscr{H}} \mathrm{d} s .
\end{aligned}
$$

Summing up the above expansion we arrive at

$$
\begin{aligned}
& f_{\alpha}(t)+g_{\alpha}(t) \\
& \quad=(56 a)+(56 b)+(59 a)+(59 b)+(59 c)+(59 d)+(60 a)+(60 b)+(60 c)+(60 d) .
\end{aligned}
$$

In the remainder of the proof we separately estimate each summand on the right side. This is readily done using basic inequalities in combination with Lemmas 2.1 and 2.2. At the end, we conclude by applying Gronwall's inequality. 
Term (56a). In the first boundary term from the integration by parts we have

$$
(56 \mathrm{a})=-i \alpha^{-1}\left\langle\psi_{\alpha}(t)-\xi_{\alpha}(t),\left.R \phi(\delta G .) \xi_{\alpha}(t)\right|_{\mathscr{H}}\right.
$$

since $R \xi_{\alpha}(t)=0$. Using the Cauchy-Schwarz inequality we obtain

$$
|(56 \mathrm{a})| \leq \frac{1}{4}\left\|\psi_{\alpha}(t)-\xi_{\alpha}(t)\right\|_{\mathscr{H}}^{2}+\alpha^{-2}\left\|R \phi(\delta G .) \xi_{\alpha}(t)\right\|_{\mathscr{H}}^{2},
$$

and with (34) and (40),

$$
\left\|R \phi(\delta G .) \xi_{\alpha}(t)\right\|_{\mathscr{H}}^{2} \leq C\left\|(N+1)^{1 / 2} \xi_{\alpha}(t)\right\|_{\mathscr{H}}^{2} \leq C \exp \left(c|t| \alpha^{-2}\right)
$$

Terms (59a) and (60a). For the other two boundary terms we proceed similarly and find

$$
\begin{aligned}
|(59 \mathrm{a})| & \leq \frac{1}{4}\left\|\psi_{\alpha}(t)-\xi_{\alpha}(t)\right\|_{\mathscr{H}}^{2}+\alpha^{-4}\left\|R \phi(\delta G .) R \phi(\delta G .) \xi_{\alpha}(t)\right\|_{\mathscr{H}} \\
& \leq \frac{1}{4}\left\|\psi_{\alpha}(t)-\xi_{\alpha}(t)\right\|_{\mathscr{H}}^{2}+C \alpha^{-4} \exp \left(c|t| \alpha^{-2}\right)
\end{aligned}
$$

as well as

$$
\begin{aligned}
|(60 \mathrm{a})| & \leq \frac{1}{4}\left\|\psi_{\alpha}(t)-\xi_{\alpha}(t)\right\|_{\mathscr{H}}^{2}+\alpha^{-6}\left\|R \phi(\delta G .) R \phi(\delta G .) R \phi(\delta G .) \xi_{\alpha}(t)\right\|_{\mathscr{H}} \\
& \leq \frac{1}{4}\left\|\psi_{\alpha}(t)-\xi_{\alpha}(t)\right\|_{\mathscr{H}}^{2}+C \alpha^{-6} \exp \left(c|t| \alpha^{-2}\right)
\end{aligned}
$$

where we have used (35) and (36) in combination with (40).

Term (56b). In this term we have

$$
(56 \mathrm{~b})=i \alpha^{-3} \int_{0}^{t}\left\langle\psi_{\alpha}(s)-\xi_{\alpha}(s), R\left([N, \phi(\delta G .)]+\phi(\delta G .) A^{\varphi_{0}}\right) \xi_{\alpha}(s)\right\rangle_{\mathscr{H}} \mathrm{d} s .
$$

Using (34), the third line of (36) and (40) we estimate

$$
\begin{aligned}
|(56 \mathrm{~b})| \leq & \alpha^{-2} \int_{0}^{t}\left\|\psi_{\alpha}(s)-\xi_{\alpha}(s)\right\|_{\mathscr{H}}^{2} \mathrm{~d} s \\
& +\frac{1}{2} \alpha^{-4} \int_{0}^{t}\left(\left\|[N, R \phi(\delta G .)] \xi_{\alpha}(s)\right\|_{\mathscr{H}}^{2}+\left\|R \phi(\delta G .) A^{\varphi_{0}} \xi_{\alpha}(s)\right\|_{\mathscr{H}}^{2}\right) \mathrm{d} s \\
\leq & \alpha^{-2} \int_{0}^{t}\left\|\psi_{\alpha}(s)-\xi_{\alpha}(s)\right\|_{\mathscr{H}}^{2} \mathrm{~d} s+C \alpha^{-2}\left(\exp \left(c|t| \alpha^{-2}\right)-1\right)
\end{aligned}
$$

Terms (59b) and (59c). Similarly as in the previous term,

$$
|(59 \mathrm{~b})|+|(59 \mathrm{c})| \leq \alpha^{-2} \int_{0}^{t}\left\|\psi_{\alpha}(s)-\xi_{\alpha}(s)\right\|_{\mathscr{H}}^{2} \mathrm{~d} s
$$




$$
\begin{aligned}
& +\frac{1}{2} \alpha^{-6} \int_{0}^{t}\left(\left\|[N, R \phi(\delta G .) R \phi(\delta G .)] \xi_{\alpha}(s)\right\|_{\mathscr{H}}^{2}\right. \\
& \left.+\left\|R \phi(\delta G .) R \phi(\delta G .) A^{\varphi_{0}} \xi_{\alpha}(s)\right\|_{\mathscr{H}}^{2}\right) \mathrm{d} s
\end{aligned}
$$

and thus by means of (35), (37) and (40) we obtain

$$
|(59 \mathrm{~b})|+|(59 \mathrm{c})| \leq \alpha^{-2} \int_{0}^{t}\left\|\psi_{\alpha}(s)-\xi_{\alpha}(s)\right\|_{\mathscr{H}}^{2} \mathrm{~d} s+C \alpha^{-4}\left(\exp \left(c|t| \alpha^{-2}\right)-1\right) .
$$

Term (59d). In this line we keep the real part (cf. (49)) and have

$$
\operatorname{Re}(59 \mathrm{~d})=\alpha^{-3} \int_{0}^{t} \operatorname{Im}\left\langle\psi_{\alpha}(s)-\xi_{\alpha}(s), P \phi(\delta G .) R \phi(\delta G .) R \phi(\delta G .) \xi_{\alpha}(s)\right\rangle_{\mathscr{H}} \mathrm{d} s
$$

(the imaginary part of the added expectation value is zero). The absolute value of the right side is bounded from above by

$$
\begin{aligned}
|\operatorname{Re}(59 \mathrm{~d})| \leq & \frac{1}{2} \alpha^{-2} \int_{0}^{t}\left(\left\|\psi_{\alpha}(s)-\xi_{\alpha}(s)\right\|_{\mathscr{H}}^{2}\right. \\
& \left.+\frac{1}{2} \alpha^{-4} \int_{0}^{t}\left\|P \phi(\delta G .) R \phi(\delta G .) R \phi(\delta G .) \xi_{\alpha}(s)\right\|_{\mathscr{H}}^{2}\right) \mathrm{d} s \\
\leq & \alpha^{-2} \int_{0}^{t}\left\|\psi_{\alpha}(s)-\xi_{\alpha}(s)\right\|_{\mathscr{H}}^{2} \mathrm{~d} s+C \alpha^{-2}\left(\exp \left(c|t| \alpha^{-2}\right)-1\right),
\end{aligned}
$$

where one uses (36) and (40) in the second step.

Term (60b). By means of (36) and (40) one obtains

$$
\begin{aligned}
|(60 \mathrm{~b})| & \leq \alpha^{-5} \int_{0}^{t}\left\|[N, R \phi(\delta G .) R \phi(\delta G .) R \phi(\delta G .)] \xi_{\alpha}(s)\right\| \mathrm{d} s \\
& \leq C \alpha^{-3}\left(\exp \left(c|t| \alpha^{-2}\right)-1\right) .
\end{aligned}
$$

Term (60c). In this term one can use (38) and (40) to find

$$
\begin{aligned}
|(60 \mathrm{c})| & \leq \alpha^{-5} \int_{0}^{t}\left\|R \phi(\delta G .) R \phi(\delta G .) R \phi(\delta G .) A^{\varphi_{0}} \xi_{\alpha}(s)\right\| \mathrm{d} s \\
& \leq C \alpha^{-3}\left(\exp \left(c|t| \alpha^{-2}\right)-1\right) .
\end{aligned}
$$

Term (60d). For the last term we apply (39) in combination with

$$
\left\|p \psi_{\alpha}(s)\right\|_{\mathscr{H}}=\left\|p e^{-i H_{\alpha}^{\mathrm{F}} s} \varphi_{0} \otimes W\left(\alpha f_{0}\right)^{*} \eta_{0}\right\|_{\mathscr{H}},
$$


see (42), as well as (40) and (41). This leads to

$$
\begin{aligned}
|(60 \mathrm{~d})| & \leq \alpha^{-4} \int_{0}^{t}\left|\left\langle\psi_{\alpha}(s), \phi(\delta G .) R \phi(\delta G .) R \phi(\delta G .) R \phi(\delta G .) \xi_{\alpha}(s)\right\rangle_{\mathscr{H}}\right| \mathrm{d} s \\
& \leq C \alpha^{-4} \int_{0}^{t}\left(1+\left\|p e^{-i H_{\alpha}^{\mathrm{F}} s} \varphi_{0} \otimes W\left(\alpha f_{0}\right)^{*} \eta_{0}\right\|_{\mathscr{H}}\right)\left\|(N+1)^{2} \xi_{\alpha}(s)\right\|_{\mathscr{H}} \mathrm{d} s \\
& \leq C \alpha^{-2}\left(\exp \left(c|t| \alpha^{-2}\right)-1\right) .
\end{aligned}
$$

Conclusion. In total, we have shown

$$
\left\|\psi_{\alpha}(s)-\xi_{\alpha}(s)\right\|_{\mathscr{H}}^{2} \leq C \alpha^{-2} \exp \left(c|t| \alpha^{-2}\right)+C \alpha^{-2} \int_{0}^{t}\left\|\psi_{\alpha}(s)-\xi_{\alpha}(s)\right\|_{\mathscr{H}}^{2} \mathrm{~d} s
$$

from which the claimed bound follows by the integral version of Gronwall's inequality.

\subsection{Proofs of Lemmas 2.1 and 2.2}

The main tool of the proof of Lemma 2.1 is the commutator method by Lieb and Yamazaki [22] by which one improves the behavior of the interaction at large momenta using the regularity of the electron wave function. More precisely one writes

$$
G_{x}(k)=\tilde{G}_{x}(k)-p \cdot K_{x}(k)+K_{x}(k) \cdot p
$$

with

$$
\tilde{G}_{x}(k)=G_{x}(k) \chi_{[0,1]}(|k|), \quad K_{x}(k)=\frac{k}{|k|^{2}} G_{x}(k) \chi_{(1, \infty)}(|k|),
$$

where $\chi$ denotes the characteristic function, i.e., $\chi_{A}(r)=1$ for all $r \in A \subseteq \mathbb{R}$ and $\chi_{A}(r)=0$ otherwise. The advantage of rewriting $G_{x}$ this way is that $\tilde{G}_{x}$ and $K_{x}$ are square-integrable,

$$
\sup _{x \in \mathbb{R}^{3}}\left(\left\|\tilde{G}_{x}(\cdot)\right\|_{L^{2}}+\left\|K_{x}(\cdot)\right\|_{L^{2}}\right)<\infty
$$

For the next proof let us recall the common bounds for the annihilation and creation operators,

$$
\|a(g) \Psi\| \mathscr{H} \leq\|g\|_{L^{2}}\left\|N^{1 / 2} \Psi\right\| \mathscr{H}, \quad\left\|a^{*}(g) \Psi\right\|_{\mathscr{H}} \leq\|g\|_{L^{2}}\left\|(N+1)^{1 / 2} \Psi\right\|_{\mathscr{H}}
$$

for any $g \in L^{2}\left(\mathbb{R}^{3}, \mathrm{~d} k\right)$. 
Proof of Lemma 2.1 For the proof of (34), we set $a^{\#} \in\left\{a, a^{*}\right\}$ and use (77), (79), $\left\|f_{0}\right\|_{L^{2}}<\infty$ and (80) to estimate

$$
\begin{aligned}
\left\|R a^{\#}(\delta G .) P \Psi\right\|_{\mathscr{H}} \leq & \left\|R a^{\#}\left(\tilde{G} \cdot-f_{0}\right) P \Psi\right\|_{\mathscr{H}} \\
& +\left\|R p \cdot a^{\#}(K .) P \Psi\right\|_{\mathscr{H}}+\left\|R a^{\#}(K .) \cdot p P \Psi\right\|_{\mathscr{H}} \\
\leq & C(\|R\|+\|R p\|+\|R\|\|p P\|)\left\|(N+1)^{1 / 2} \eta\right\|_{\mathcal{F}} \\
\leq & C\left\|(N+1)^{1 / 2} \eta\right\|_{\mathcal{F}},
\end{aligned}
$$

where $\|\cdot\|=\|\cdot\| \mathscr{L}$ denotes the norm on the space of bounded operators $\mathscr{L}\left(L^{2}\left(\mathbb{R}^{3}, \mathrm{~d} x\right)\right)$. That $\|R\|+\|p P\|_{L^{2}}<\infty$ is clear. To show $\|R p\|<\infty$ we compute

$$
\begin{aligned}
\|p R \psi\|_{L^{2}}^{2} & =\left\langle\psi, R\left(h^{\varphi_{0}}-\lambda\right) R \psi\right\rangle_{L^{2}}+\left\langle\psi, R\left(\lambda-V^{\varphi_{0}}\right) R \psi\right\rangle_{L^{2}} \\
& \leq\langle\psi, R \psi\rangle_{L^{2}}+\frac{1}{2}\left\langle\psi, R p^{2} R \psi\right\rangle_{L^{2}}+C\|R \psi\|_{L^{2}}^{2}
\end{aligned}
$$

where we used $\pm V^{\varphi_{0}} \leq \frac{1}{2} p^{2}+C$ as shown, e.g., in [19, Lemma III.2]. ${ }^{4}$ Since the bound (81) holds equally if $R$ is replaced by $P$ and since

$$
\left[N, \phi\left(\delta G_{x}\right)\right]=a^{*}\left(\delta G_{x}\right)-a\left(\delta G_{x}\right),
$$

this proves (34).

In order to prove (35) we derive the bound for $\| R a^{\#_{1}}\left(\delta G\right.$.) $R a^{\#_{2}}(\delta G$.) $\Psi \|$ with $a^{\#_{i}} \in\left\{a, a^{*}\right\}$. Proceeding similarly as in (81), we find

$$
\left\|R a^{\#_{1}}(\delta G .) R a^{\#_{2}}(\delta G .) \Psi\right\|_{\mathscr{H}} \leq C\left\|(N+1)^{1 / 2} R^{1 / 2} a^{\#_{2}}(\delta G .) \Psi\right\|_{\mathscr{H}} .
$$

From here we use

$$
\begin{aligned}
(N+1)^{1 / 2} R^{1 / 2} a\left(\delta G_{x}\right) \Psi & =R^{1 / 2} a\left(\delta G_{x}\right) N^{1 / 2} \Psi \\
(N+1)^{1 / 2} R^{1 / 2} a^{*}\left(\delta G_{x}\right) \Psi & =R^{1 / 2} a^{*}\left(\delta G_{x}\right)(N+2)^{1 / 2} \Psi,
\end{aligned}
$$

together with

$$
\left\|R^{1 / 2} a^{\#_{2}}(\delta G .) \Psi\right\|_{\mathscr{H}} \leq C\left\|(N+1)^{1 / 2} \eta\right\|_{\mathcal{F}}
$$

The latter is obtained in complete analogy to (81). The bounds for the other terms on the 1.h.s. of (35) are derived the same way. Since the derivation of (36) and (37) is also very similar, we omit further details.

To prove (38) we proceed again as in (81) and find

$$
\begin{aligned}
& \left|\left\langle\Phi, a^{\#_{1}}(\delta G .) R a^{\#_{2}}(\delta G .) R a^{\#_{3}}(\delta G .) R a^{\#_{4}}(\delta G .) \Psi\right\rangle_{\mathscr{H}}\right| \\
& \quad \leq\|\Phi\|_{\mathscr{H}}\left\|\left(a^{\#_{1}}\left(\tilde{G} .-f_{0}\right)-a^{\#_{1}}(K .) \cdot p\right) R a^{\#_{2}}(\delta G .) R a^{\#_{3}}(\delta G .) R a^{\#_{4}}(\delta G .) \Psi\right\|_{\mathscr{H}}
\end{aligned}
$$

${ }^{4}$ Note that our potential $V^{\varphi_{0}}$ coincides (up to a factor) with $V_{\varphi}$ for $\varphi=f_{0} \in L^{2}\left(\mathbb{R}^{3}, \mathrm{~d} k\right.$ ) in [19]. 


$$
\begin{aligned}
& +\left|\left\langle\Phi, p \cdot a^{\#_{1}}(K .) R a^{\#_{2}}(\delta G .) R a^{\#_{3}}(\delta G .) R a^{\#_{4}}(\delta G .) \Psi\right\rangle_{\mathscr{H}}\right| \\
\leq & C(1+\|p \Phi\| \mathscr{H})\left\|(N+1)^{1 / 2} R^{1 / 2} a^{\#_{2}}(\delta G .) R a^{\#_{3}}(\delta G .) R^{1 / 2} a^{\#_{4}}(\delta G .) \Psi\right\|_{\mathscr{H}} .
\end{aligned}
$$

By estimating the last factor similarly as the right hand side of (84) we obtain (38).

Proof of Lemma 2.2 We start by verifying the following bound,

$$
\left|\left\langle\eta,(N+1)^{j-1}\left[N, A^{\varphi_{0}}\right](N+1)^{m-j} \eta\right\rangle_{\mathcal{F}}\right| \leq C\left\|(N+1)^{m / 2} \eta\right\|_{\mathcal{F}}^{2}
$$

for $1 \leq j \leq m$. To do so, use (83) to write

$$
\left[N, A^{\varphi_{0}}\right]=\left\langle\varphi_{0},\left(a^{*}(G .)-a(G .)\right) R\left(a^{*}(G .)+a(G .)\right) \varphi_{0}\right\rangle+\text { h.c. },
$$

and then estimate each term separately. We illustrate the argument for the term $A_{++}^{\varphi_{0}}=$ $\left\langle\varphi_{0}, a^{*}(G .) R a^{*}(G .) \varphi_{0}\right\rangle_{L^{2}}$ for which we have

$$
\begin{aligned}
&\langle\eta,\left.(N+1)^{j-1} A_{++}^{\varphi_{0}}(N+1)^{m-j} \eta\right\rangle_{\mathcal{F}} \\
& \quad=\left\{\begin{array}{lll}
\left\langle\eta,(N+1)^{j-1}(N-1)^{\frac{m}{2}+1-j} A_{++}^{\varphi_{0}}(N+1)^{\frac{m}{2}-1} \eta\right\rangle_{\mathcal{F}} & \left(\frac{m}{2}+1 \geq j\right), \\
\left\langle\eta,(N+1)^{\frac{j+i}{2}-1} A_{++}^{\varphi_{0}}(N+3)^{\frac{j-i}{2}}(N+1)^{m-j} \eta\right\rangle_{\mathcal{F}}, & \text { with } i=m+2-j \quad\left(j \geq \frac{m}{2}+1\right) .
\end{array}\right.
\end{aligned}
$$

Taking the absolute value and using the Cauchy-Schwarz inequality we can bound the first line from above by

$$
\left\|(N+1)^{j-1}(N-1)^{\frac{m}{2}+1-j} \eta\right\|_{\mathcal{F}}\left\|A_{++}^{\varphi_{0}}(N+1)^{\frac{m}{2}-1} \eta\right\|_{\mathcal{F}} \leq C\left\|(N+1)^{\frac{m}{2}} \eta\right\|_{\mathcal{F}}^{2}
$$

where we used $\left\|A_{++}^{\varphi_{0}} \eta\right\|_{\mathcal{F}} \leq C\|(N+1) \eta\|_{\mathcal{F}}, \eta \in \mathcal{F}$, which is proved the same way as the bound for the left side of (84). Similarly we find the following upper bound for the second line,

$$
\left\|(N+1)^{\frac{j+i}{2}-1} \eta\right\|_{\mathcal{F}}\left\|A_{++}^{\varphi_{0}}(N+3)^{\frac{j-i}{2}}(N+1)^{m-j} \eta\right\|_{\mathcal{F}} \leq C\left\|(N+1)^{\frac{m}{2}} \eta\right\|_{\mathcal{F}}^{2} .
$$

Repeating the same argument for the other terms in (90) leads to the stated bound in (89).

Next let $\eta_{\alpha}(t)=\exp \left(-i \alpha^{-2}\left(N-A^{\varphi_{0}}\right) t\right) \eta$ and compute the time derivative

$$
\frac{\mathrm{d}}{\mathrm{d} t}\left\langle\eta_{\alpha}(t), N^{m} \eta_{\alpha}(t)\right\rangle_{\mathcal{F}}=-\alpha^{-2} \sum_{j=1}^{m}\left\langle\eta_{\alpha}(t), N^{j-1} i\left[N, A^{\varphi_{0}}\right] N^{m-j} \eta_{\alpha}(t)\right\rangle_{\mathcal{F}}
$$

which for $m \in\{1,2,3,4,5\}$ is easily checked explicitly. Setting $z(t)=\sum_{j=1}^{5} \|(N+$ $1)^{j / 2} \eta_{\alpha}(t) \|_{\mathcal{F}}^{2}$, we have by (89) and (93), $\left|\frac{\mathrm{d}}{\mathrm{d} t} z(t)\right| \leq C \alpha^{-2} z(t)$. Since $\sup _{\alpha>0} z(0)<$ $\infty$ by assumption, it follows from Gronwall's inequality that $z(t) \leq C \exp \left(c|t| \alpha^{-2}\right)$. 
For a proof of (41), let $\psi_{\alpha}(t)=e^{-i H_{\alpha}^{\mathrm{F}} t} \varphi_{0} \otimes W\left(\alpha f_{0}\right)^{*} \eta$ and estimate

$$
\begin{aligned}
\left\|p \psi_{\alpha}(t)\right\|_{\mathscr{H}}^{2} & \leq C\left\langle\psi_{\alpha}(t),\left(H_{\alpha}^{\mathrm{F}}+1\right) \psi_{\alpha}(t)\right\rangle_{\mathscr{H}}=C\left\langle\psi_{\alpha}(0),\left(H_{\alpha}^{\mathrm{F}}+1\right) \psi_{\alpha}(0)\right\rangle_{\mathscr{H}} \\
& =C\left(1+\mathcal{E}^{\mathrm{P}}\left(\varphi_{0}\right)+\alpha^{-2}\langle\eta, N \eta\rangle_{\mathcal{F}}\right)
\end{aligned}
$$

for some constant $C>0$. Here we used $N \geq 0$ and $p^{2}+\alpha^{-2} N \leq C\left(H_{\alpha}^{\mathrm{F}}+1\right)$ in the first step (see, e.g., [13, Lemma A.5]) and the commutation relation (42) together with $\left\langle\varphi_{0}, \delta G_{x} \varphi_{0}\right\rangle_{L^{2}}=0$ in the third step.

\subsection{Proofs of Proposition 1.1 and Corollaries 1.3 and 1.4}

Proof of proposition 1.1 The identity in (16) follows from $\mathcal{E}^{\mathrm{P}}\left(\varphi_{0}\right)=\lambda+\left\|f_{0}\right\|_{L^{2}}^{2}$ together with the commutation relations (44) and (45). That $\mathscr{D}(N) \subseteq \mathscr{D}\left(N-A^{\varphi_{0}}\right)$ follows from

$$
\left\|A^{\varphi_{0}} \eta\right\|_{\mathcal{F}}=\left\|P \phi(G .) R \phi(G .) \varphi_{0} \otimes \eta\right\|_{\mathscr{H}} \leq C\|(N+1) \eta\|_{\mathcal{F}}
$$

which is proven the same way as the bound for the 1.h.s. of (84). Using (83) one further finds

$$
\left|\left\langle\eta,\left[A^{\varphi_{0}}, N\right] \eta\right\rangle_{\mathcal{F}}\right| \leq C\langle\eta, N \eta\rangle_{\mathcal{F}}
$$

for all $\eta \in \mathcal{F}_{0}$ with $\mathcal{F}_{0} \subseteq \mathcal{F}$ denoting the dense subspace of all Fock space vectors that have only finitely many nonzero components. Since $\mathcal{F}_{0}$ is a core of the number operator $N$, we can infer that $N-A^{\varphi_{0}}$ is essentially self-adjoint by a variant of Nelson's commutator theorem [5, Corollary 1.1]. Alternatively one could conclude self-adjointness of $N-A^{\varphi_{0}}$ from the criteria for self-adjointness of Fock space operators found in [4].

In the following two proofs we make use of the bound

$$
\left.\operatorname{Tr}_{\mathscr{H}_{1}}\left|\operatorname{Tr}_{\mathscr{H}_{2}}\right| \Psi\right\rangle\left\langle\Phi|| \leq\|\Psi\| \mathscr{H}_{1} \otimes \mathscr{H}_{2}\|\Phi\|_{\mathscr{H}_{1} \otimes \mathscr{H}_{2}}\right.
$$

where $\mathscr{H}_{1}, \mathscr{H}_{2}$ are two separable Hilbert spaces and $\Psi, \Phi \in \mathscr{H}_{1} \otimes \mathscr{H}_{2}$. The inequality follows from the variational characterization of the trace. For a proof see [8, Appendix D].

Proof of Corollary 1.3 We recall $\Psi_{\alpha}(t)=e^{-i H_{\alpha}^{\mathrm{F}} t} \varphi_{0} \otimes W\left(\alpha f_{0}\right)^{*} \eta_{0}$ and write

$$
\begin{aligned}
\left|\Psi_{\alpha}(t)\right\rangle\left\langle\Psi_{\alpha}(t)|=|\right. & \left|e^{-i H_{\alpha}^{\varphi_{0}} t} \Psi_{\alpha}(0)\right\rangle\left\langle e^{-i H_{\alpha}^{\varphi_{0}} t} \Psi_{\alpha}(0)\right| \\
& +\left|\Psi_{\alpha}(t)\right\rangle\left\langle\left(e^{-i H_{\alpha}^{\mathrm{F}} t}-e^{-i H_{\alpha}^{\varphi_{0}} t}\right) \Psi_{\alpha}(0)\right| \\
& +\left|\left(e^{-i H_{\alpha}^{\mathrm{F}} t}-e^{-i H_{\alpha}^{\varphi_{0}} t}\right) \Psi_{\alpha}(0)\right\rangle\left\langle e^{-i H_{\alpha}^{\varphi_{0}} t} \Psi_{\alpha}(0)\right| .
\end{aligned}
$$


Since $e^{-i H_{\alpha}^{\varphi_{0}} t}$ acts nontrivially only on the Fock space,

$$
\operatorname{Tr}_{\mathcal{F}}\left|e^{-i H_{\alpha}^{\varphi_{0}} t} \Psi_{\alpha}(0)\right\rangle\left\langle e^{-i H_{\alpha}^{\varphi_{0}} t} \Psi_{\alpha}(0)|=| \varphi_{0}\right\rangle\left\langle\varphi_{0}\right|
$$

Applying (97) to the two last terms in (98) thus leads to

$$
\left.\operatorname{Tr}_{L^{2}}\left|\operatorname{Tr}_{\mathcal{F}}\right| \Psi_{\alpha}(t)\right\rangle\left\langle\Psi_{\alpha}(t)|-| \varphi_{0}\right\rangle\left\langle\varphi_{0}|| \leq 2||\left(e^{-i H_{\alpha}^{\mathrm{F}} t}-e^{-i H_{\alpha}^{\varphi_{0}} t}\right) \Psi_{\alpha}(0) \|_{\mathscr{H}} .\right.
$$

Together with Theorem 1.2 this proves the corollary.

Proof of Corollary 1.4 For $E \in \mathbb{R}$ we write

$$
\begin{aligned}
& \left|W\left(\alpha f_{0}\right) \Psi_{\alpha}(t)\right\rangle\left\langle W\left(\alpha f_{0}\right) \Psi_{\alpha}(t)\right| \\
& \left.=\mid \varphi_{0} \otimes e^{-i \alpha^{-2}\left(N-A^{\varphi_{0}}\right) t} \eta_{0}\right)\left\langle\varphi_{0} \otimes e^{-i \alpha^{-2}\left(N-A^{\varphi_{0}}\right) t} \eta_{0}\right| \\
& \quad+\left|W\left(\alpha f_{0}\right) \Psi_{\alpha}(t)\right\rangle\left\langle W\left(\alpha f_{0}\right) \Psi_{\alpha}(t)-\varphi_{0} \otimes e^{-i \alpha^{-2}\left(N-A^{\varphi_{0}}+E\right) t} \eta_{0}\right| \\
& \quad+\left|W\left(\alpha f_{0}\right) \Psi_{\alpha}(t)-\varphi_{0} \otimes e^{-i \alpha^{-2}\left(N-A^{\varphi_{0}}+E\right) t} \eta_{0}\right\rangle\left\langle\varphi_{0} \otimes e^{-i \alpha^{-2}\left(N-A^{\varphi_{0}}+E\right) t} \eta_{0}\right| .
\end{aligned}
$$

From here we proceed similarly as in the proof of Corollary 1.3. Taking the partial trace in the first line gives

$$
\begin{aligned}
& \operatorname{Tr}_{L^{2}}\left|\varphi_{0} \otimes e^{-i \alpha^{-2}\left(N-A^{\varphi_{0}}\right) t} \eta_{0}\right\rangle\left\langle\varphi_{0} \otimes e^{-i \alpha^{-2}\left(N-A^{\varphi_{0}}\right) t} \eta_{0}\right| \\
& \quad=\mid e^{-i \alpha^{-2}\left(N-A^{\left.\varphi_{0}\right) t} \eta_{0}\right\rangle\left\langle e^{-i \alpha^{-2}\left(N-A^{\left.\varphi_{0}\right) t}\right.} \eta_{0}\right| .}
\end{aligned}
$$

Using (97) for the second and third line then leads to

$$
\begin{aligned}
& \operatorname{Tr}_{\mathcal{F}}\left.\left|\operatorname{Tr}_{L^{2}}\right| W\left(\alpha f_{0}\right) \Psi_{\alpha}(t)\right\rangle\left\langle W\left(\alpha f_{0}\right) \Psi_{\alpha}(t)\right| \\
&-\left|e^{-i \alpha^{-2}\left(N-A^{\varphi_{0}}\right) t} \eta_{0}\right\rangle\left\langle e^{-i \alpha^{-2}\left(N-A^{\left.\varphi_{0}+\right) t} \eta_{0}||\right.}\right. \\
& \quad \leq 2|| W\left(\alpha f_{0}\right) \Psi_{\alpha}(t)-\varphi_{0} \otimes e^{-i \alpha^{-2}\left(N-A^{\varphi_{0}}+E\right) t} \eta_{0} \|_{\mathscr{H}}
\end{aligned}
$$

Setting $E=\mathcal{E}^{\mathrm{P}}\left(\varphi_{0}\right)$ we can employ (16) and use the unitarity of $W\left(\alpha f_{0}\right)$ to see that the last line equals

$$
2\left\|W\left(\alpha f_{0}\right)\left(\Psi_{\alpha}(t)-e^{-i H_{\alpha}^{\varphi_{0}} t} \Psi_{\alpha}(0)\right)\right\|_{\mathscr{H}}=2\left\|\left(e^{-i H_{\alpha}^{\mathrm{F}} t}-e^{-i H_{\alpha}^{\varphi} t}\right) \Psi_{\alpha}(0)\right\|_{\mathscr{H}}
$$

The corollary now follows from Theorem 1.2 together with the identity

$$
\left|e^{-i \alpha^{-2}\left(N-A^{\varphi_{0}}\right) t} \eta_{0}\right\rangle\left\langle e^{-i \alpha^{-2}\left(N-A^{\varphi_{0}}\right) t} \eta_{0}|=| U_{\mathcal{V}_{\alpha}(t)} \eta_{0}\right\rangle\left\langle U_{\mathcal{V}_{\alpha}(t)} \eta_{0}\right|
$$


which we shall prove below.

Proof of (105). For $\varepsilon=\int_{\mathbb{R}^{3}}(2 \pi|k|)^{-2}\left\|R^{1 / 2} e^{-i k x} \varphi_{0}\right\|_{L^{2}}^{2} \mathrm{~d} k$ it holds that

$$
\exp \left(-i \alpha^{-2}\left(N-A^{\varphi_{0}}+\varepsilon\right) t\right) \eta_{0}=U_{\mathcal{V}_{\alpha}(t)} \eta_{0}
$$

To show this we follow closely the argument from [2, Lem. 2.8 and App. B] where a similar identity was proven in the context of the dynamics of weakly interacting bosons. The argument is based on well-known facts about Bogoliubov transformations and quasi-free states and a general result about the dynamics generated by quadratic Hamiltonians [23, Prop. 7].

At this point it is useful to introduce the pointwise annihilation and creation operators $a_{k}, a_{k}^{*}$ defined by the requirement that

$$
a(g)=\int_{\mathbb{R}^{3}} \overline{g(k)} a_{k} \mathrm{~d} k, \quad a^{*}(g)=\int_{\mathbb{R}^{3}} g(k) a_{k}^{*} \mathrm{~d} k
$$

for any $g \in L^{2}\left(\mathbb{R}^{3}, \mathrm{~d} k\right)$. The commutation relations (3) now read

$$
\left[a_{k}, a_{l}^{*}\right]=\delta(k-l), \quad\left[a_{k}, a_{l}\right]=\left[a_{k}^{*}, a_{l}^{*}\right]=0 \quad \forall k, l \in \mathbb{R}^{3} .
$$

Using (17), (107) and (108) a short computation leads to

$$
N-A^{\varphi_{0}}+\varepsilon=\mathrm{d} \Gamma(1-\mathcal{G})-\frac{1}{2} \int_{\mathbb{R}^{3}} \int_{\mathbb{R}^{3}}\left(\mathcal{K}(k, l) a_{k}^{*} a_{l}^{*}+\overline{\mathcal{K}(k, l)} a_{k} a_{l}\right) \mathrm{d} k \mathrm{~d} l
$$

where $\mathrm{d} \Gamma(1-\mathcal{G})$ denotes the second quantization of the one-body operator $1-\mathcal{G}$, i.e.,

$$
\mathrm{d} \Gamma(1-\mathcal{G})=\int_{\mathbb{R}^{3}} \int_{\mathbb{R}^{3}}(\delta(k-l)-\mathcal{G}(k, l)) a_{k}^{*} a_{l} \mathrm{~d} k \mathrm{~d} l,
$$

see (25) and (26) for a definition of $\mathcal{K}(k, l)$ and $\mathcal{G}(k, l)$, respectively. To the operator on the right side of (109) we can apply [23, Prop. 7]. The requirements of this proposition are satisfied since $1-\mathcal{G}: L^{2}\left(\mathbb{R}^{3}, \mathrm{~d} k\right) \rightarrow L^{2}\left(\mathbb{R}^{3}, \mathrm{~d} k\right)$ is bounded and $\mathcal{K}: L^{2}\left(\mathbb{R}^{3}, \mathrm{~d} k\right) \rightarrow L^{2}\left(\mathbb{R}^{3}, \mathrm{~d} k\right)$ is a Hilbert-Schmidt operator which can be verified by means of (77). By part (iii) of [23, Prop. 7] it follows in particular that for any quasifree state $\eta_{0} \in \mathcal{F}$, the time-evolved state $\eta_{\alpha}(t)=\exp \left(-i \alpha^{-2}\left(N-A^{\varphi_{0}}+\varepsilon\right) t\right) \eta_{0}$ is again quasi-free (the bound $\left\langle\eta_{\alpha}(t), N \eta_{\alpha}(t)\right\rangle \leq C \exp \left(c|t| \alpha^{-2}\right)$ can be checked directly by means of Gronwall's inequality). It is further not difficult to verify that the state $U_{\mathcal{V}_{\alpha}(t)} \eta_{0}$ is also quasi-free $\left(\eta_{0}=U_{\mathcal{W}} \Omega_{0}\right.$ for some Bogoliubov map $\mathcal{W}$ and thus $U_{\mathcal{V}_{\alpha}(t)} \eta_{0}=U_{\mathcal{V}_{\alpha}(t) \circ \mathcal{W}} \Omega_{0}$ with Bogoliubov map $\left.\mathcal{V}_{\alpha}(t) \circ \mathcal{W}\right)$. To show equality between the quasi-free states $\eta_{\alpha}(t)$ and $U_{\mathcal{V}_{\alpha}(t)} \eta_{0}$ we compare their reduced one-body density matrices. This is sufficient because of the well-known fact that quasifree states are uniquely determined by their reduced one-body density matrices. For $\xi \in \mathcal{F}$ the reduced one-body density matrices $\gamma_{\xi}: L^{2}\left(\mathbb{R}^{3}, \mathrm{~d} k\right) \rightarrow L^{2}\left(\mathbb{R}^{3}, \mathrm{~d} k\right)$ and $\alpha_{\xi}: L^{2}\left(\mathbb{R}^{3}, \mathrm{~d} k\right) \rightarrow L^{2}\left(\mathbb{R}^{3}, \mathrm{~d} k\right)$ are defined by 


$$
\left\langle f, \gamma_{\xi} g\right\rangle_{L^{2}}=\left\langle\xi, a^{*}(g) a(f) \xi\right\rangle_{\mathcal{F}}, \quad\left\langle f, \alpha_{\xi} \bar{g}\right\rangle_{L^{2}}=\langle\xi, a(g) a(f) \xi\rangle_{\mathcal{F}}
$$

for all $f, g \in L^{2}\left(\mathbb{R}^{3}, \mathrm{~d} k\right)$. In order to show $\gamma_{\eta_{\alpha}(t)}=\gamma_{U \mathcal{V}_{\alpha}(t) \eta_{0}}$ and $\alpha_{\eta_{\alpha}(t)}=\alpha_{U \mathcal{V}_{\alpha}(t) \eta_{0}}$ we argue that they solve the same pair of differential equations with the same initial condition $\gamma_{\eta_{0}}$ and $\alpha_{\eta_{0}}$, respectively, and then use that the solution to this pair of differential equations is unique (the latter was shown in [23, Prop. 7]).

Instead of computing the time derivative of $\gamma_{\eta_{\alpha}(t)}$ and $\alpha_{\eta_{\alpha}(t)}$, and similarly for $U_{\mathcal{V}_{\alpha}(t)} \eta_{0}$, it is more convenient to determine the time derivative of $\left\langle\eta_{\alpha}(t)\right.$, $\left.A\left(F_{1}\right) A\left(F_{2}\right) \eta_{\alpha}(t)\right\rangle_{\mathcal{F}}$ with $A(F)$ the generalized annihilation operator as defined above (21). For $F_{1}, F_{2} \in L^{2}\left(\mathbb{R}^{3}, \mathrm{~d} k\right) \oplus L^{2}\left(\mathbb{R}^{3}, \mathrm{~d} k\right)$ we have

$$
i \frac{\mathrm{d}}{\mathrm{d} t}\left\langle\eta_{\alpha}(t), A\left(F_{1}\right) A\left(F_{2}\right) \xi_{\alpha}(t)\right\rangle_{\mathcal{F}}=\alpha^{-2}\left\langle\eta_{\alpha}(t),\left.\left[N-A^{\varphi_{0}}, A\left(F_{1}\right) A\left(F_{2}\right)\right] \xi_{\alpha}(t)\right|_{\mathcal{F}}\right.
$$

and it follows by a straightforward computation that

$$
\left[N-A^{\varphi_{0}}, A\left(F_{1}\right) A\left(F_{2}\right)\right]=A\left(\mathcal{A} F_{1}\right) A\left(F_{2}\right)+A\left(F_{1}\right) A\left(\mathcal{A} F_{2}\right)
$$

with

$$
\mathcal{A}=\left(\begin{array}{cc}
1-\mathcal{G} & \mathcal{K} \\
-\overline{\mathcal{K}} & -1+\overline{\mathcal{G}}
\end{array}\right)
$$

Next we use $U_{\mathcal{V}}^{*} A(F) U_{\mathcal{V}}=A\left(\mathcal{V}^{-1} F\right)$, cf. (23), to obtain

$$
\left\langle U_{\mathcal{V}_{\alpha}(t)} \eta_{0}, A\left(F_{1}\right) A\left(F_{2}\right) U_{\mathcal{V}_{\alpha}(t)} \eta_{0}\right\rangle_{\mathcal{F}}=\left\langle\eta_{0}, A\left(\mathcal{V}_{\alpha}^{-1}(t) F_{1}\right) A\left(\mathcal{V}_{\alpha}^{-1}(t) F_{2}\right) \eta_{0}\right\rangle_{\mathcal{F}}
$$

By means of $\left(i \partial_{t} \mathcal{V}_{\alpha}^{-1}(t)\right) \mathcal{V}_{\alpha}(t)=-\mathcal{V}_{\alpha}^{-1}(t)\left(i \partial_{t} \mathcal{V}_{\alpha}(t)\right)$ together with $i \partial_{t} \mathcal{V}_{\alpha}(t)=$ $\alpha^{-2} \mathcal{A} \mathcal{V}_{\alpha}(t)$, we can compute the time derivative

$$
\begin{aligned}
i \frac{\mathrm{d}}{\mathrm{d} t} & \left\langle\eta_{0}, A\left(\mathcal{V}_{\alpha}^{-1}(t) F_{1}\right) A\left(\mathcal{V}_{\alpha}^{-1}(t) F_{2}\right) \eta_{0}\right\rangle_{\mathcal{F}} \\
& =\left\langle\eta_{0},\left(A\left(-i \partial_{t} \mathcal{V}_{\alpha}^{-1}(t) F_{1}\right) A\left(\mathcal{V}_{\alpha}^{-1}(t) F_{2}\right)+A\left(\mathcal{V}_{\alpha}^{-1}(t) F_{1}\right) A\left(-i \partial_{t} \mathcal{V}_{\alpha}^{-1}(t) F_{2}\right)\right) \eta_{0}\right\rangle_{\mathcal{F}} \\
& =\alpha^{-2}\left\langle\eta_{0},\left(A\left(\mathcal{V}_{\alpha}^{-1}(t) \mathcal{A} F_{1}\right) A\left(\mathcal{V}_{\alpha}^{-1}(t) F_{2}\right)+A\left(\mathcal{V}_{\alpha}^{-1}(t) F_{1}\right) A\left(\mathcal{V}_{\alpha}^{-1}(t) \mathcal{A} F_{2}\right)\right) \eta_{0}\right\rangle_{\mathcal{F}} \\
& =\alpha^{-2}\left\langle U_{\mathcal{V}_{\alpha}(t)} \eta_{0},\left(A\left(\mathcal{A} F_{1}\right) A\left(F_{2}\right)+A\left(F_{1}\right) A\left(\mathcal{A} F_{2}\right)\right) U_{\mathcal{V}_{\alpha}(t)} \eta_{0}\right\rangle_{\mathcal{F}}
\end{aligned}
$$

Comparing (112) and (113) with (115) and (116) we see that the pairs of reduced one-body density matrices $\left(\gamma_{\eta_{\alpha}(t)}, \alpha_{\eta_{\alpha}(t)}\right)$ and $\left(\gamma_{U_{\mathcal{V}_{\alpha}(t)} \eta_{0}}, \alpha_{U_{\mathcal{V}_{\alpha}(t)} \eta_{0}}\right)$ solve the same differential equation. Since the solution to this equation is unique, see [23, Prop. 7], and since $\eta_{\alpha}(0)=U_{\mathcal{V}_{\alpha}(0)} \eta_{0}=\eta_{0}$, we conclude their equality. This implies $\eta_{\alpha}(t)=U_{\mathcal{V}_{\alpha}(t)} \eta_{0}$ and hence proves the claimed identity. 
Acknowledgements I thank Marcel Griesemer for many interesting discussions about the Fröhlich polaron and also for valuable comments on this manuscript. Helpful discussions with Nikolai Leopold and Robert Seiringer are also gratefully acknowledged. This work was partially supported by the Deutsche Forschungsgemeinschaft $(D F G)$ through the Research Training Group 1838: Spectral Theory and Dynamics of Quantum Systems.

Funding Open Access funding enabled and organized by Projekt DEAL.

Open Access This article is licensed under a Creative Commons Attribution 4.0 International License, which permits use, sharing, adaptation, distribution and reproduction in any medium or format, as long as you give appropriate credit to the original author(s) and the source, provide a link to the Creative Commons licence, and indicate if changes were made. The images or other third party material in this article are included in the article's Creative Commons licence, unless indicated otherwise in a credit line to the material. If material is not included in the article's Creative Commons licence and your intended use is not permitted by statutory regulation or exceeds the permitted use, you will need to obtain permission directly from the copyright holder. To view a copy of this licence, visit http://creativecommons.org/licenses/by/4.0/.

\section{References}

1. Alexandrov, A.S., Devreese, J.T.: Advances in Polaron Physics. Springer, New York (2010)

2. Boßmann, L., Petrat, S., Pickl, P., Soffer, A.: Beyond Bogoliubov Dynamics. Preprint arXiv:1912.11004 (2019)

3. Donsker, M.D., Varadhan, S.R.S.: Asymptotics for the polaron. Commun. Pure Appl. Math. 36, 505528 (1983)

4. Falconi, M.: Self-adjointness criterion for operators in Fock spaces. Math. Phys. Anal. Geom. 18, Art. $2(2015)$

5. Faris, W.G., Lavine, R.B.: Commutators and self-adjointness of Hamiltonian operators. Commun. Math. Phys. 35, 39-48 (1974)

6. Feliciangeli, D., Rademacher, S., Seiringer, R.: Persistence of the spectral gap for the Landau-Pekar equations. Lett. Math. Phys. 111, 19 (2021)

7. Frank, R.L., Schlein, B.: Dynamics of a strongly coupled polaron. Lett. Math. Phys. 104, 911-929 (2014)

8. Frank, R.L., Gang, Z.: Derivation of an effective evolution equation for a strongly coupled polaron. Anal. PDE 10, 379-422 (2017)

9. Frank, R.L., Gang, Z.: A non-linear adiabatic theorem for the Landau-Pekar equations. Oberwolfach Rep. (2017). https://doi.org/10.1016/j.jfa.2020.108631

10. Frank, R.L., Gang, Z.: A non-linear adiabatic theorem for the one-dimensional Landau-Pekar equations. J. Funct. Anal. 279, 7 (2020)

11. Frank, R.L., Seiringer, R.: Quantum corrections to the Pekar asymptotics of a strongly coupled polaron. Commun. Pure Appl. Math. (2020). https://doi.org/10.1002/cpa.21944

12. Fröhlich, H.: Theory of electrical breakdown in ionic crytals. Proc. R. Soc. Lond. A 160, 230-241 (1937)

13. Griesemer, M.: On the dynamics of polarons in the strong-coupling limit. Rev. Math. Phys. 29(10), 1750030 (2017)

14. Griesemer, M., Wünsch, A.: Self-adjointness and domain of the Fröhlich Hamiltonian. J. Math. Phys. 57(2), (2016)

15. Jeblick, M., Mitrouskas, D., Petrat, S., Pickl, P.: Free time evolution of a tracer particle coupled to a fermi gas in the high-density limit. Commun. Math. Phys. 356, 143-187 (2017)

16. Jeblick, M., Mitrouskas, D., Pickl, P.: Effective dynamics of two tracer particles coupled to a fermi gas in the high-density limit. In: Macroscopic Limits of Quantum Systems, Springer Proceedings in Mathematics \& Statistics (2017)

17. Lampart, J., Schmidt, J.: On Nelson-type hamiltonians and abstract boundary conditions. Commun. Math. Phys. 367, 629-663 (2019) 
18. Leopold, N., Mitrouskas, D., Rademacher, S., Schlein, B., Seiringer, R.: Landau-Pekar equations and quantum fluctuations for the dynamics of a strongly coupled polaron. Preprint. arXiv:2005.02098 (2020)

19. Leopold, N., Rademacher, S., Schlein, B., Seiringer, R.: The Landau-Pekar equations: adiabatic theorem and accuracy. Preprint. arXiv:1904.12532, Analysis \& PDE (in press)

20. Lieb, E.H.: Existence and uniqueness of the minimizing solution of Choquard's nonlinear equation. Stud. Appl. Math. 57, 93-105 (1977)

21. Lieb, E.H., Thomas, L.E.: Exact ground state energy of the strong-coupling polaron. Commun. Math. Phys. 183, 511-519 (1997). Erratum: ibid. 188, 499 (1997)

22. Lieb, E.H., Yamazaki, K.: Ground-state energy and effective mass of the polaron. Phys. Rev. 111, 728-733 (1958)

23. Nam, P.T., Napiórkowski, M.: Bogoliubov correction to the mean-field dynamics of interacting bosons. Adv. Theor. Math. Phys. 21, 683-738 (2017)

24. Pekar, S.I.: Untersuchung über die Elektronentheorie der Kristalle. Berlin. Verlag, Akad (1954)

25. Reed, M., Simon, B.: Methods of Modern Mathematical Physics, Analysis of Operators, vol. IV. Academic Press, New York (1978)

26. Reed, M., Simon, B.: Methods of Modern Mathematical Physics. Functional Analysis, vol. I. Academic Press, New York (1980)

27. Solovej, J.P.: Many body quantum mechanics. Lecture notes. https://www.mathematik.uni-muenchen. de/ sorensen/Lehre/SoSe2013/MQM2/skript.pdf (2007)

Publisher's Note Springer Nature remains neutral with regard to jurisdictional claims in published maps and institutional affiliations. 